\title{
On the definition of mass in general relativity: Noether charges and conserved quantities in diffeomorphism invariant theories
}

\author{
Brian P. Dolan* \\ Department of Theoretical Physics, Maynooth University, Maynooth, Co. Kildare W23 F2H6, Ireland \\ and School of Theoretical Physics, Dublin Institute for Advanced Studies, \\ 10 Burlington Road, Dublin D04 C932, Ireland
}

(Received 31 May 2018; published 3 August 2018)

\begin{abstract}
Geometrically the phase space of a mechanical system involves the cotangent bundle of the configuration space. The phase space of a relativistic field theory is infinite dimensional and can be endowed with a symplectic structure defined in a perfectly covariant manner that is very useful for discussing symmetries and conserved quantities of the system. In general relativity the symplectic structure takes the Darboux form, and it is shown in this work that the presence of a cosmological constant does not change this conclusion. For space-times that admit timelike Killing vectors the formalism can be used to define mass in general relativity, and it is known that, for asymptotically flat black holes, this mass is identical to the usual Arnowitt-Desner-Misner mass while for asymptotically anti-de Sitter Kerr metrics it is the same as the Henneaux-Teitelboim mass. We show that the same formalism can also be used to derive the Brown-York mass and the Bondi mass for stationary space times, in particular the Brown-York mass has a natural interpretation in terms of differential forms on the space of solutions of the theory.
\end{abstract}

DOI: $10.1103 /$ PhysRevD.98.044010

\section{INTRODUCTION}

Some years ago Crnković and Witten [1] gave a method for constructing a symplectic form on the space of solutions, $\mathcal{S}$, of the equations of motion of a relativistic field theory. They used their formalism to obtain the relevant symplectic forms for Yang-Mills theory and for general relativity. Their construction provides a covariant description of relativistic field theories in the phase space of solutions modulo gauge transformations (and diffeomorhpisms) $\mathcal{G}, \widehat{\mathcal{S}}=\mathcal{S} / \mathcal{G}$, which is ideally suited to studying symmetries and conserved quantities.

The idea of a symplectic structure for diffeomorphism invariant theories was first introduced in [2] to investigate instabilities in rotating relativistic fluids. Wald and Collaborators have generalized the Crnković and Witten's formalism to a very wide class of diffeomorphism invariant theories in [3-6] and studied conserved quantities associated with Killing symmetries, such as angular momentum in rotationally invariant solutions and mass in stationary solutions.

We first summarize the construction of the symplectic form and the role of diffeomorphisms and Killing symmetries in general. Examples of the statements made in the Introduction are given in the main text following. One starts with an $(n+1)$-dimensional space-time manifold $\mathcal{M}$ with boundary $\partial M$. The space-time comes with a metric, and

*bdolan@thphys.nuim.ie possibly other fields such as Yang-Mills fields, and the space of all field configurations $\mathcal{F}$ is infinite dimensional. The dynamics are determined by a variational principle with a Lagrangian $L$, which is a gauge invariant $(n+1)$ form on $\mathcal{M}$, and an action

$$
\mathcal{A}[\mathcal{F}]=\int_{\mathcal{M}} L(\mathcal{F})
$$

which is a diffeomorphism invariant functional of the fields. A solution of the equations of motion is a field configuration that extremizes $\mathcal{A}$.

It is assumed that space-time can be foliated using a time parameter $t$ and that surfaces of constant $t$ are spacelike hypersurfaces, $\Sigma_{t}$. An infinitesimal variation of any solution of the equations of motion that satisfies the linearized equations of motion is a 1 -form on $\mathcal{S}$, more correctly a cross section of the cotangent bundle $T^{*} \mathcal{S}$.

A symplectic form on the space of solutions is obtained by using $L$ to construct an 1 -form ${ }^{1} \boldsymbol{\theta}$ on $\mathcal{S}$, which is also an $n$-form on $\mathcal{M}$, and is a presymplectic potential on $\Sigma_{t}$, i.e., $\boldsymbol{\theta}$ does not itself furnish a symplectic potential on the space of solutions (it is not necessarily diffeomorphism invariant) but it gives one when diffeomorphisms are modded out. When $\Sigma_{t}$ is a Cauchy surface,

\footnotetext{
${ }^{1}$ In this general discussion bold face symbols will represent forms on $\mathcal{S}$.
} 


$$
\Theta=\int_{\Sigma_{t}} \boldsymbol{\theta}
$$

gives a presymplectic potential on $\mathcal{S}$. Under a second independent variation of the dynamical fields one obtains another $n$-form on $\mathcal{M}$

$$
\omega=\delta \theta,
$$

where $\delta$ is the exterior derivative on the space of solution $\mathcal{S}$. Since $\boldsymbol{\delta}^{2}=0$

$$
\delta \omega=0,
$$

and $\boldsymbol{\omega}$ is a presymplectic density in the sense that

$$
\boldsymbol{\Omega}=\int_{\Sigma_{t}} \boldsymbol{\omega}
$$

is a presymplectic 2 -form on $\mathcal{S}{ }^{2}$ It is called presymplectic because it is not a genuine symplectic density, it is not necessarily gauge and diffeomorphism invariant.

There is a very elegant interplay between the $d$-cohomology on $\mathcal{M}$ and the $\delta$-cohomology on $\mathcal{S}$. The construction is such that $\omega$ is not only closed as a 1 -form on $\mathcal{S}$ but also as an $n$-form on $\mathcal{M}, d \omega=0$; hence

$$
\int_{\mathcal{M}} d \omega=\int_{\partial \mathcal{M}} \omega=0
$$

If $\mathcal{M}$ has the topology $T \times \Sigma$, where $T=\left[t, t^{\prime}\right] \subset R$ is a time interval and $\Sigma$ is a compact Cauchy hypersurface without boundary, then $\partial \mathcal{M}$ consists of 2 copies of $\Sigma$, Fig. 1. Then foliate $\mathcal{M}$, using $t$ as a time-parameter, and $\int_{\Sigma_{t}} \omega$ is independent of the value of $t$ chosen so we can drop the subscript $t$ and

$$
\mathbf{\Omega}=\int_{\Sigma} \omega
$$

is independent of the Cauchy hypersurface $\Sigma$.

Under a projection from the space of solutions $\mathcal{S}$ to the space of solutions modulo gauge transformations and diffeomorphisms $\widehat{\mathcal{S}}$ the symplectic form $\widehat{\boldsymbol{\Omega}}$ on $\widehat{\mathcal{S}}$ must pull-back to the presymplectic form $\boldsymbol{\Omega}$ on $\mathcal{S}$. This will be the case if

(i) $\omega$ is gauge invariant and $d$-exact whenever one of the metric variations is a diffeomorphism;

(ii) $\Sigma$ is compact without boundary.

Then it is shown in [5] that, when one of the metric variations corresponds to a diffeomorphism generated by a vector field $\vec{X}$, the dependence of $\omega$ on $\vec{X}$ is such that $\omega(\vec{X})$

\footnotetext{
${ }^{2} \boldsymbol{\delta} \int_{\Sigma_{t}}=\int_{\Sigma_{t}} \boldsymbol{\delta}$ since, while $\Sigma_{t}$ depends on the coordinates, it is independent of the fields, in particular of the metric.
}

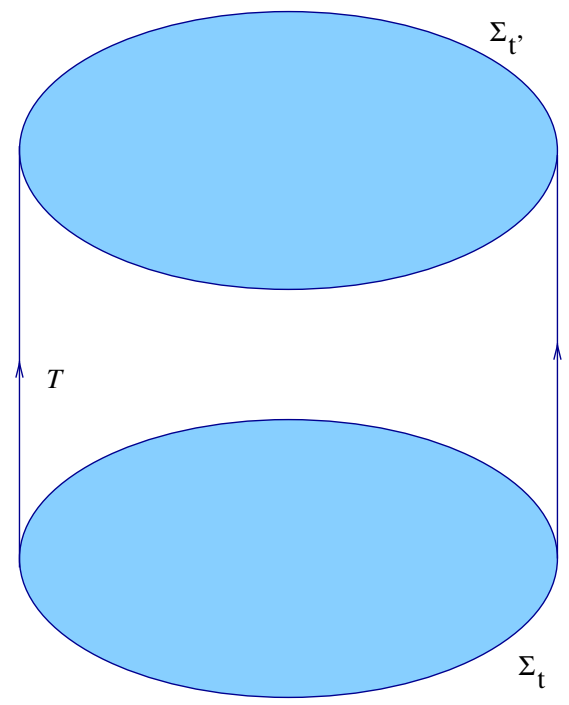

FIG. 1. The boundary of $\mathcal{M}$ consists of two spacelike hypersurfaces $\Sigma$ and $\Sigma^{\prime}$ connected by a timelike tube $T$, with $T=$ $\partial \Sigma \times\left[t, t^{\prime}\right]$.

is not only $d$-closed as an $n$-form on $\mathcal{M}$ but it is also $d$-exact $^{3}$

$$
\omega(\vec{X})=d \phi(\vec{X})
$$

for some $(n-1)$-form $\phi(\vec{X})$. If $\Sigma$ is compact without boundary,

$$
\boldsymbol{\Omega}[\vec{X}]=\int_{\Sigma} \boldsymbol{\omega}(\vec{X})=0
$$

when one of the variations is a diffeomorphism. Under the projection $\mathcal{S} \rightarrow \widehat{\mathcal{S}}$ the symplectic form $\widehat{\mathbf{\Omega}}$ on $\widehat{\mathcal{S}}$ then pulls back to the presymplectic form $\boldsymbol{\Omega}$ on $\mathcal{S}$, [1,3].

If $\Sigma$ has a boundary $\partial \Sigma$ then we can use (1) to deduce that

$$
\boldsymbol{\Omega}[\vec{X}]=\int_{\Sigma} \boldsymbol{\omega}(\vec{X})=\int_{\partial \Sigma} \boldsymbol{\phi}(\vec{X}) .
$$

Provided $\int_{\partial \Sigma} \boldsymbol{\phi}(\vec{X})$ vanishes whenever one of the field variations is due to a diffeomorphism then $\boldsymbol{\Omega}$ is again a genuine presymplectic form. This will be the case e.g., if the vector field $\vec{X}$ generating the diffeomorphism vanishes fast enough on $\partial \Sigma$.

Furthermore if the diffeomorphism $\vec{X}=\vec{K}$ corresponds to a Killing symmetry of the solution then $\omega(\vec{K})=d \phi(\vec{K})$ vanishes identically and $\int_{\partial \Sigma} \boldsymbol{\phi}(\vec{K})=0$, even when $\vec{K}$ does not vanish on the boundary [6]. If $\partial \Sigma$ consists of two disconnected pieces, $\partial \Sigma=\partial \Sigma_{1} \cup \partial \Sigma_{2}$ (Fig. 2), then the

\footnotetext{
${ }^{3}$ The condition $\boldsymbol{\delta} \boldsymbol{\theta}=d \boldsymbol{\phi}$ is reminiscent of the Stora-Zumino descent equations in the study of anomalies [7]. Indeed the whole formalism is intimately related to a cohomological structure that fits naturally into a double complex [8].
} 


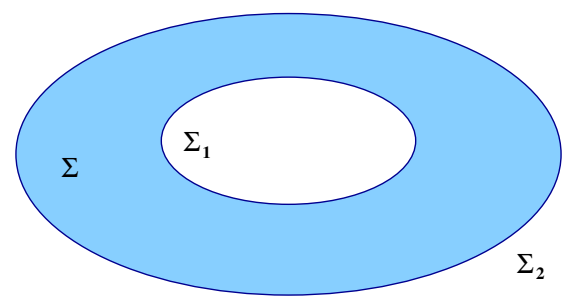

FIG. 2. When $\partial \Sigma=\partial \Sigma_{2} \cup \partial \Sigma_{1}$, and $\vec{K}$ is Killing, $\Phi[\vec{K}]$ is independent of which connected piece of the boundary it is evaluated on, $\partial \Sigma_{1}$ or $\partial \Sigma_{2} . \boldsymbol{\Phi}[\vec{K}]$ depends on the fields and a variation thereof but if one segment of the boundary, $\partial \Sigma_{1}$ say, is fixed we can distort $\partial \Sigma_{2}$ and move it around, provided it does not pass through a singularity in the geometry $\boldsymbol{\Phi}[\vec{K}]$ evaluated on $\Sigma_{2}$ does not change.

integral over each piece must be equal and opposite and they cancel. With suitable orientations

$$
\boldsymbol{\Phi}[\vec{K}]=\int_{\partial \Sigma_{1}} \boldsymbol{\phi}(\vec{K})=\int_{\partial \Sigma_{2}} \boldsymbol{\phi}(\vec{K})
$$

can be nonzero. If one of the pieces, e.g., $\partial \Sigma_{1}$, is held fixed then $\boldsymbol{\Phi}[\vec{K}]$ evaluated on $\partial \Sigma_{2}$ is independent of the $(n-1)$ dimensional surface $\partial \Sigma_{2} . \Sigma$ does not have to be a Cauchy surface for this statement to be true.

If $\boldsymbol{\Phi}[\vec{K}]$ is $\boldsymbol{\delta}$-exact, and only if it is $\boldsymbol{\delta}$-exact, then

$$
\boldsymbol{\Phi}[\vec{K}]=\delta Q[\vec{K}]
$$

for some $Q[\vec{K}]$. This $Q[\vec{K}]$ is a charge related to a Hamiltonian associated with the flow generated by $\vec{K}$, [5].

Among the key ingredients to generate conserved quantities from this construction are a general coordinate invariant action and a solution of the equations of motion with a Killing vector generating the symmetry. This formalism was shown in [6] to reproduce the ADM mass [9] for stationary asymptotically flat black holes in Einstein gravity. At the same time it clarifies the origin of the mysterious factor of 2 that is well-known to arise when comparing the Komar mass with the ADM mass [5,10,11]. The construction in [3] is general enough to include theories with a cosmological constant $\Lambda$, of either sign when $\Sigma$ is compact without boundary. When $\Sigma$ has a boundary we can restrict to negative $\Lambda$ so that the asymptotic regime of a black hole solution is well-defined, and it was shown in $[12,13]$ that Lee and Wald's formalism correctly reproduces the Henneaux-Teitelboim mass $[14,15]$ for an asymptotically AdS Kerr black hole.

In this work we shall explore the formalism of Lee and Wald [3] and Wald [5] in some detail and show how it relates to some other definitions of mass in the literature, not just the ADM mass but also the Brown-York mass [16] and the Bondi mass [17] in stationary space-times. For example we shall see that the Brown-York mass, which is expressed as the difference of two extrinsic curvatures, can be viewed as a 1-form on $\mathcal{S}$. The formalism also reproduces the Bondi mass when applied to asymptotically flat spacetimes using Bondi-Sachs coordinates.

The general formalism has a very nice mathematical structure of a double complex[18], which is the natural mathematical language for describing cohomology. This formal structure is described elsewhere [8].

Section II reviews Wald's general construction in the context of Einstein gravity with a cosmological constant. The exposition is given in terms of differential forms on $\mathcal{M}$ and $\mathcal{S}$ as this is the most natural framework for treating differential cohomology. In Sec. III the symplectic 2-form is derived for nonzero $\Lambda$ and shown to be of Darboux form, extending the results of [3] to include a cosmological constant. Section IV, in which conserved quantities associated with a timelike Killing vector are discussed, contains our main results. We derive an exact result for the variation of the mass in Einstein gravity, valid at any distance outside a stationary gravitating mass. This includes the asymptotically flat case in Sec. IV A where the expression simplifies and asymptotically reproduces the ADM mass, as derived in [6]. We also relate Wald's expression to the extrinsic curvature of $\partial \Sigma$ and the Brown-York mass [16]. It usually stated that the ADM mass in asymptotically flat spacetimes is completely equivalent to that defined by Brown and York, and a proof is given in [19]; in the formalism presented here these two masses are only the same if the falloff conditions on the metric are slightly stronger than those usually assumed. The Bondi mass is also derived using Wald's formalism.

Finally the conclusions are summarized in Sec. V. Some more technical details are relegated to a number of Appendices.

\section{EINSTEIN GRAVITY}

We shall focus on the Einstein action $\mathcal{A}$ with a cosmological constant on a space-time $\mathcal{M}$. We keep the dimension of space, $n$, general for the moment and will specialize to $n=3$ later. In units with $G=c=1$ the Lagrangian density is

$$
L=\frac{1}{16 \pi}\left(R_{a b} \wedge * e^{a b}-2 \Lambda * 1\right),
$$

where

$$
R_{a b}=d \omega_{a b}+\omega_{a c} \wedge \omega_{b}^{c}
$$

are the curvature 2-forms, $e^{a}$ are orthonormal 1-forms (vielbeins), $e^{a b}=e^{a} \wedge e^{b}$ denotes the wedge product and $*$ is the Hodge star operator. In second order formulation the connection 1-forms $\omega_{a b}$ are determined in terms of the orthonormal 1-forms using the torsion free condition

$$
D e^{a}=d e^{a}+\omega_{a b} \wedge e^{b}=0 .
$$


The corresponding action is

$$
\mathcal{A}=\frac{1}{16 \pi} \int_{\mathcal{M}}\left(R_{a b} \wedge * e^{a b}-2 \Lambda * 1\right),
$$

and the equations of motion are

$$
E^{c}=\frac{1}{16 \pi}\left(R_{a b} \wedge * e^{a b c}-2 \Lambda * e^{c}\right)=0,
$$

equivalent to

$$
\mathcal{R}_{a b}=\Lambda \eta_{a b},
$$

where $\mathcal{R}_{a b}$ are the components of the Ricci tensor in an orthonormal basis and $\eta_{a b}=\operatorname{diag}(-1,+1, \ldots,+1)$.

Under an infinitesimal variation $e^{a} \rightarrow e^{a}+\boldsymbol{\delta} e^{a}$ the linearized equations of motion, with constant $\Lambda$, are

$$
\delta \mathcal{R}_{a b}=0,
$$

and the variation in the Lagrangian density is

$$
\delta L=d \boldsymbol{\theta}
$$

with

$$
d \boldsymbol{\theta}=\frac{1}{16 \pi}\left(\boldsymbol{\delta} R_{a b}\right) \wedge * e^{a b}=\frac{1}{16 \pi}(\boldsymbol{\delta} \mathcal{R}) * 1,
$$

where the equations of motion have been used and $\mathcal{R}=$ $\mathcal{R}^{a}{ }_{a}$ is the Ricci scalar. We shall refer to field configurations that satisfy the equations of motion together with variations that satisfy the linearized equations of motion as "on shell".

Now

$$
\left(\boldsymbol{\delta} R_{a b}\right) \wedge * e^{a b}=D\left(\boldsymbol{\delta} \omega_{a b}\right) \wedge * e^{a b}=d\left(\boldsymbol{\delta} \omega_{a b} \wedge * e^{a b}\right)
$$

so

$$
\boldsymbol{\theta}=\frac{1}{16 \pi}\left(\boldsymbol{\delta} \omega_{a b}\right) \wedge * e^{a b} \bmod d
$$

and

$$
\boldsymbol{\omega}=-\frac{1}{16 \pi}\left(\boldsymbol{\delta} \omega_{a b}\right) \bar{\Lambda}\left(\boldsymbol{\delta} * e^{a b}\right) \bmod d
$$

(the symbol $\bar{\Lambda}$ here represents both the wedge product on $\mathcal{M}$ and on $\mathcal{S}$ simultaneously, we hope that the distinction between the presymplectic density $\omega$ and the connection 1 -forms $\omega_{a b}$ is clear).

The variation $\delta e^{a}$ can itself be expanded in the orthonormal basis as

$$
\boldsymbol{\delta} e^{a}=\boldsymbol{\delta}\left(e^{a}{ }_{\mu} d x^{\mu}\right)=\left(\boldsymbol{\delta} e^{a}{ }_{\mu}\right) d x^{\mu}:=\boldsymbol{\Delta}^{a}{ }_{b} e^{b},
$$

where $x^{\mu}, \mu=0,1, \ldots, n$, are coordinates on $\mathcal{M}$ and

$$
\boldsymbol{\Delta}^{a}{ }_{b}=\left(\boldsymbol{\delta} e^{a}{ }_{\mu}\right)\left(e^{-1}\right)_{b}^{\mu}
$$

is a $(n+1) \times(n+1)$ matrix. Not all such variations actually correspond to changing the metric. Decomposing

$$
\boldsymbol{\Delta}_{a b}=\eta_{a c} \boldsymbol{\Delta}_{b}^{c}
$$

into symmetric and antisymmetric parts

$$
\boldsymbol{\Delta}_{a b}=\boldsymbol{S}_{a b}+\boldsymbol{A}_{a b},
$$

with $\boldsymbol{S}_{a b}=\boldsymbol{S}_{b a}$ and $\boldsymbol{A}_{a b}=-\boldsymbol{A}_{b a}$, only $\boldsymbol{S}_{a b}$ can change the metric, $\boldsymbol{A}_{a b}$ merely generate local Lorentz transformations ${ }^{4}$ under which $L$ is invariant.

Furthermore not all $\boldsymbol{S}_{a b}$ correspond to real changes in the metric, under a diffeomorphism $\vec{X}$

$$
\boldsymbol{S}_{a b}=\frac{1}{2}\left(D_{a} \boldsymbol{X}_{b}+D_{b} \boldsymbol{X}_{a}\right) .
$$

As $\boldsymbol{\delta} \omega_{a b}$ is linear in $\boldsymbol{\delta} e^{a}$ the decomposition (13) implies a similar decomposition for $\delta \omega_{a b}$. Using the torsion free condition (A2)

$$
\boldsymbol{\delta} \omega_{a b}=\left(D_{b} \boldsymbol{S}_{a c}-D_{a} \boldsymbol{S}_{b c}-D_{c} \boldsymbol{A}_{a b}\right) e^{c} .
$$

This means that $\left(\boldsymbol{\delta} \omega_{a b}\right) \wedge * e^{a b}$ is not gauge invariant; however

$$
\left(D \boldsymbol{A}_{a b}\right) \wedge * e^{a b}=d\left(\boldsymbol{A}_{a b} * e^{a b}\right)=d *\left(e^{a} \wedge \boldsymbol{\delta} e_{a}\right)
$$

is $d$-exact, and we can use the arbitrariness in (8) to define

$\boldsymbol{\theta}\left(e^{a}, \boldsymbol{\delta} e^{a}\right)=\frac{1}{16 \pi}\left\{\left(\boldsymbol{\delta} \omega_{a b}\right) \wedge * e^{a b}+d *\left(e^{a} \wedge \boldsymbol{\delta} e_{a}\right)\right\}$,

which is gauge invariant by construction. In terms of $\boldsymbol{S}_{a b}$ and the covariant derivative $D_{a}$

$$
\boldsymbol{\theta}\left(e^{a}, \boldsymbol{\delta} e^{a}\right)=\frac{1}{8 \pi}\left(D_{b} \boldsymbol{S}_{a}{ }^{b}-\partial_{a} \boldsymbol{S}_{b}{ }^{b}\right) * e^{a} .
$$

The explicit form of $\omega\left(e^{e}, \delta_{1} e^{a}, \delta_{2} e^{a}\right)$ in terms of $\left(\Delta_{1}\right)^{a}{ }_{b}=$ $\delta_{1} e^{a}{ }_{\mu}\left(e^{-1}\right)^{\mu}{ }_{b}$ and $\left(\Delta_{2}\right)^{a}{ }_{b}=\delta_{2} e^{a}{ }_{\mu}\left(e^{-1}\right)^{\mu}{ }_{b}$ is not very illuminating but for completeness is given in Appendix B. Here we just remark that, since it is gauge invariant, it only depends on the symmetric variations $\left(\boldsymbol{S}_{1}\right)_{a b}=\frac{1}{2}\left\{\left(\boldsymbol{\Delta}_{1}\right)_{a b}+\right.$ $\left.\left(\boldsymbol{\Delta}_{1}\right)_{b a}\right\}$ and $\left(\boldsymbol{S}_{2}\right)_{a b}=\frac{1}{2}\left\{\left(\boldsymbol{\Delta}_{2}\right)_{a b}+\left(\boldsymbol{\Delta}_{2}\right)_{b a}\right\}$.

Note that (16) has no explicit dependence on the cosmological constant $\Lambda$, though there is an implicit dependence when $e^{a}$ are on shell. This is to be expected from (2) as the cosmological term in the action only involves $e^{a}$, not their derivatives, and so cannot influence $d \boldsymbol{\theta}$ in (6). In particular the presence of a nonzero $\Lambda$ does not affect the symplectic form and the statement by Lee and Wald in [3] that the symplectic form takes the Darboux form is unchanged when $\Lambda$ is introduced, as we shall see explicitly in Sec. III.

\footnotetext{
${ }^{4}$ From now on we shall use the term "gauge transformations" for such local Lorentz transformations, as distinct from diffeomorphisms.
} 
Diffeomorphisms are generated by an infinitesimal vector field $\vec{X}$

$$
\delta e^{a}=\mathcal{L}_{\vec{X}} e^{a}=d i_{\vec{X}} e^{a}+i_{\vec{X}} d e^{a} .
$$

When the variation is a diffeomorphism one finds, for the Einstein action (4),

$$
\boldsymbol{\theta}\left(e^{a}, \mathcal{L}_{\vec{X}} e^{a}\right)=-\frac{1}{16 \pi}\left(2 e^{a} \wedge * D D \boldsymbol{X}_{a}+d * d \boldsymbol{X}\right),
$$

where $\boldsymbol{X}=\boldsymbol{X}_{a} e^{a}$ is both a 1 -form on $\mathcal{M}$ and on $\mathcal{S}{ }^{5}$ Now $D D \boldsymbol{X}_{a}=R_{a b} \boldsymbol{X}^{b}$ leading to

$$
\begin{aligned}
\boldsymbol{\theta}\left(e^{a}, \mathcal{L}_{\overrightarrow{\boldsymbol{X}}} e^{a}\right) & =\frac{1}{16 \pi}\left(2 \mathcal{R}_{a b} \boldsymbol{X}^{a} * e^{b}-d * d \boldsymbol{X}\right) \\
& =\frac{1}{16 \pi}(2 \Lambda * \boldsymbol{X}-d * d \boldsymbol{X}),
\end{aligned}
$$

where the equations of motion have been used in the last step. But on shell

$$
L=\frac{\Lambda}{8 \pi} * 1
$$

and by definition $* X=i_{\vec{X}} * 1$, so

$$
\boldsymbol{\theta}\left(e^{a}, \mathcal{L}_{\overrightarrow{\boldsymbol{X}}} e^{a}\right)=\boldsymbol{i}_{\overrightarrow{\boldsymbol{X}}} L+\boldsymbol{J}(\overrightarrow{\boldsymbol{X}}),
$$

where

$$
\boldsymbol{J}(\overrightarrow{\boldsymbol{X}})=-\frac{1}{16 \pi} d * d \boldsymbol{X}
$$

and $\boldsymbol{J}(\overrightarrow{\boldsymbol{X}})=d \boldsymbol{Q}$ is $d$-exact, with

$$
\boldsymbol{Q}=-\frac{1}{16 \pi} * d \boldsymbol{X}
$$

$d \boldsymbol{Q}$ is a 2-form on $\mathcal{M}$. The similarity between $d \boldsymbol{Q}=d * d \vec{X}$ with the vacuum Maxwell equations was observed in [20].

The symplectic density is obtained from

$$
\begin{aligned}
\boldsymbol{\omega}(\overrightarrow{\boldsymbol{X}}) & =\boldsymbol{\delta} \boldsymbol{\theta}\left(e^{a}, \mathcal{L}_{\overrightarrow{\boldsymbol{X}}} e^{a}\right)+\mathcal{L}_{\vec{X}} \boldsymbol{\theta}\left(e^{a}, \boldsymbol{\delta} e^{a}\right) \\
& =\boldsymbol{\delta}\left(\boldsymbol{i}_{\overrightarrow{\boldsymbol{X}}} L-\frac{1}{16 \pi} d * d \boldsymbol{X}\right)+\left(d \boldsymbol{i}_{\overrightarrow{\boldsymbol{X}}}+\boldsymbol{i}_{\overrightarrow{\boldsymbol{X}}} d\right) \boldsymbol{\theta}\left(e^{a}, \boldsymbol{\delta} e^{a}\right) \\
& =d\left(-\frac{1}{16 \pi} \boldsymbol{\delta}(* d \boldsymbol{X})+\boldsymbol{i}_{\overrightarrow{\boldsymbol{X}}} \boldsymbol{\theta}\left(e^{a}, \boldsymbol{\delta} e^{a}\right)\right),
\end{aligned}
$$

where we have used

$$
\delta \boldsymbol{i}_{\vec{X}} L=-i_{\vec{X}} \boldsymbol{\delta} L=-i_{\vec{X}} d \boldsymbol{\theta}\left(e^{a}, \boldsymbol{\delta} e^{a}\right) .
$$

\footnotetext{
${ }^{5}$ In practice we need not take $\vec{X}$ to be infinitesimal. Since all subsequent formulas are linear in $\vec{X}$ we can rescale $\vec{X} \rightarrow \epsilon \vec{X}$, with $\epsilon \ll 1$, and $\epsilon$ is just an overall factor in all formulas. Indeed we can let $\boldsymbol{\epsilon}$ represent a constant 1-form on $\mathcal{S}$, so that $\boldsymbol{\delta} \boldsymbol{\epsilon}=-\boldsymbol{\epsilon} \boldsymbol{\delta}$ and $\vec{X}=\epsilon \vec{X}$ is a vector on $\mathcal{M}$ and a 1 -form on $\mathcal{S}$, with $i_{\vec{X}}=\epsilon i_{\vec{X}}$, [8].
}

We have proven that

$$
\boldsymbol{\omega}\left(e^{a}, \mathcal{L}_{\overrightarrow{\boldsymbol{X}}} e^{a}, \boldsymbol{\delta} e^{a}\right)=d \phi(\overrightarrow{\boldsymbol{X}})
$$

with

$$
\boldsymbol{\phi}(\overrightarrow{\boldsymbol{X}})=-\frac{1}{16 \pi} \boldsymbol{\delta} * d \boldsymbol{X}+\boldsymbol{i}_{\overrightarrow{\boldsymbol{X}}} \boldsymbol{\theta}\left(e^{a}, \boldsymbol{\delta} e^{a}\right)
$$

and

$$
\boldsymbol{\Omega}[\overrightarrow{\boldsymbol{X}}]=\int_{\Sigma} \boldsymbol{\omega}(\overrightarrow{\boldsymbol{X}})=\int_{\partial \Sigma} \phi(\overrightarrow{\boldsymbol{X}}),
$$

where $\boldsymbol{\Omega}[\overrightarrow{\boldsymbol{X}}]=\boldsymbol{\Omega}\left[e^{a}, \boldsymbol{L}_{\overrightarrow{\boldsymbol{X}}} e^{a}, \boldsymbol{\delta} e^{a}\right]$. When $\Sigma$ has no boundary $\boldsymbol{\Omega}[\overrightarrow{\boldsymbol{X}}]$ vanishes and a general $\boldsymbol{\Omega}$ will be a genuine pull back from $\widehat{\boldsymbol{\Omega}}$ on $\widehat{\mathcal{S}}$. If $\Sigma$ has a boundary this is still the case provided $\vec{X}$ is constrained to vanish on the boundary. ${ }^{6}$

If $\vec{X}=\vec{K}$ is Killing then $S_{a b}=0$, and not only does $\boldsymbol{\Omega}[\overrightarrow{\boldsymbol{K}}]$ vanish but $\boldsymbol{\omega}(\overrightarrow{\boldsymbol{K}})$ is identically zero, see (B2), independently of any boundary conditions or choice of hypersurfaces. This is an important observation: on shell $\boldsymbol{\omega}\left(e^{a}, \mathcal{L}_{\overrightarrow{\boldsymbol{K}}} e^{a}, \boldsymbol{\delta} e^{a}\right)=\boldsymbol{\omega}(\overrightarrow{\boldsymbol{K}})$ vanishes identically for any Killing vector $\vec{K}$. Since $\boldsymbol{\omega}(\overrightarrow{\boldsymbol{X}})=d \phi(\overrightarrow{\boldsymbol{X}})$ this implies that

$$
\boldsymbol{\phi}\left(e^{a}, \overrightarrow{\boldsymbol{K}}, \boldsymbol{\delta} e^{a}\right)=-\frac{1}{16 \pi} \boldsymbol{\delta} * d \boldsymbol{K}+\boldsymbol{i}_{\overrightarrow{\boldsymbol{K}}} \boldsymbol{\theta}\left(e^{a}, \boldsymbol{\delta} e^{a}\right)
$$

is $d$-closed, but it need not vanish and can carry useful information.

If $\vec{K}$ is purely tangential to $\partial \Sigma$, e.g., if $\vec{K}$ generates rotations about the origin and $\partial \Sigma$ is the sphere at infinity, then

$$
\int_{\partial \Sigma} i_{\vec{K}} \theta=0
$$

and

$$
0=\boldsymbol{\Omega}[\overrightarrow{\boldsymbol{K}}]=-\boldsymbol{\delta}\left(\frac{1}{16 \pi} \int_{\partial \Sigma} * d \boldsymbol{K}\right)
$$

so

$$
\frac{1}{16 \pi} \int_{\partial \Sigma} * d \boldsymbol{K}
$$

is invariant under on shell perturbations of the metric. If the boundary $\partial \Sigma$ consists of disconnected pieces, e.g., if $\partial \Sigma=\partial \Sigma_{1} \cup \partial \Sigma_{2}$ consists of two separate pieces $\partial \Sigma_{1}$ and $\partial \Sigma_{2}$, then define

$$
\boldsymbol{\Phi}_{p}[\overrightarrow{\boldsymbol{K}}]=-\frac{1}{16 \pi} \int_{\partial \Sigma_{p}} \boldsymbol{\delta} * d \boldsymbol{K}
$$

$(p=1,2)$ and, with appropriate orientations,

${ }^{6}$ If $\partial \Sigma$ is some asymptotic regime with unbounded area then this statement must be qualified, $\vec{X}$ must vanish "fast enough" on the boundary. 


$$
\boldsymbol{\Omega}[\overrightarrow{\boldsymbol{K}}]=\boldsymbol{\Phi}_{2}\left[\overrightarrow{\boldsymbol{K}}, \boldsymbol{\delta} e^{a}\right]-\boldsymbol{\Phi}_{1}\left[\overrightarrow{\boldsymbol{K}}, \boldsymbol{\delta} e^{a}\right]=0 .
$$

We can deform $\Sigma$ and move either of the boundaries around, keeping the other fixed-provided we do not pass through any singularities in the geometry and

$$
\boldsymbol{\Phi}\left[\overrightarrow{\boldsymbol{K}}, \boldsymbol{\delta} e^{a}\right]:=\boldsymbol{\Phi}_{1}\left[\overrightarrow{\boldsymbol{K}} a, \boldsymbol{\delta} e^{a}\right]=\boldsymbol{\Phi}_{2}\left[\overrightarrow{\boldsymbol{K}}, \boldsymbol{\delta} e^{a}\right]
$$

is unchanged by such deformations. This statement is independent of any symplectic structure, and $\Sigma$ need not be a Cauchy surface. $\boldsymbol{\Phi}$ in (24) will not change as the boundary of $\Sigma$ is moved around, as long as it does not move through a region containing matter or a singularity in the geometry.

$$
\boldsymbol{Q}[\overrightarrow{\boldsymbol{K}}]=-\frac{1}{16 \pi} \int_{\partial \Sigma_{1}} * d \boldsymbol{K}=-\frac{1}{16 \pi} \int_{\partial \Sigma_{2}} * d \boldsymbol{K}
$$

is the integral of the Komar 2-form associated with the Killing vector $\vec{K}$ [10], it is not itself invariant under metric perturbations of course.

When $\vec{K}$ is not purely tangential to $\partial \Sigma$ however $\boldsymbol{\theta}$ can contribute to $\phi$ and the story is more involved, but we can still define a charge if $\boldsymbol{i}_{\overrightarrow{\boldsymbol{K}}} \boldsymbol{\theta}$ is $\boldsymbol{\delta}$-exact. In the most general case we have

$$
\boldsymbol{\Phi}_{p}[\overrightarrow{\boldsymbol{K}}]=-\frac{1}{16 \pi} \int_{\partial \Sigma_{p}} \boldsymbol{\delta} * d \boldsymbol{K}+\int_{\partial \Sigma_{p}} \boldsymbol{i}_{\overrightarrow{\boldsymbol{K}}} \boldsymbol{\theta}\left(e^{a}, \boldsymbol{\delta} e^{a}\right) .
$$

If furthermore $\boldsymbol{i}_{\overrightarrow{\boldsymbol{K}}} \boldsymbol{\theta}$ is $\boldsymbol{\delta}$-exact, so $\boldsymbol{\phi}=\boldsymbol{\delta} \boldsymbol{\rho}(\overrightarrow{\boldsymbol{K}})$ for some $\boldsymbol{\rho}$, then

$$
\boldsymbol{Q}[\overrightarrow{\boldsymbol{K}}]=\int_{\partial \Sigma_{1}} \boldsymbol{\rho}(\overrightarrow{\boldsymbol{K}})=\int_{\partial \Sigma_{2}} \boldsymbol{\rho}(\overrightarrow{\boldsymbol{K}})
$$

is a candidate for a Noether charge ${ }^{7}$ associated with the symmetry generated by $\vec{K} . Q[\overrightarrow{\boldsymbol{K}}]$ and $\boldsymbol{\rho}(\overrightarrow{\boldsymbol{K}})$ are 1 -forms on $\mathcal{S}$ but only through their dependence on $\boldsymbol{K}$, they do not themselves involve a metric variation but also are not invariant under genuine metric perturbations. Examples are given in Sec. IV A for $\vec{K}=\frac{\partial}{\partial t}$ a timelike Killing vector, in which case $\mathcal{Q}\left[\frac{\partial}{\partial t}\right]$ is a mass.

\section{THE SYMPLECTIC FORM}

We shall now explicitly calculate the symplectic form for Einstein gravity with a cosmological constant $\Lambda$. The case $\Lambda=0$ was analyzed in $[1,3,6]$.

Assume the space-time $\mathcal{M}$ can be foliated into spacelike hypersurfaces $\Sigma_{t}$ of constant $t$. Defining lapse and shift

\footnotetext{
${ }^{7}$ This is not in general the same as the Noether charge associated the entropy, as defined in [5].
}

functions $N$ and $N^{\alpha}$ for the foliation in the usual way we can choose the orthonormal 1 -forms ${ }^{8}$

$$
e^{a}=e_{\mu}^{a} d x^{\mu}
$$

to decompose as

$$
e^{0}=N d t \quad \text { and } \quad e^{i}=\widetilde{e}^{i}+\frac{N^{i}}{N} e^{0}
$$

where

$$
\widetilde{e}^{i}=\widetilde{e}_{\alpha}^{i} d x^{\alpha}
$$

and $N^{i}=\widetilde{e}^{i}{ }_{\alpha} N^{\alpha}$. With (27) we have made a partial choice of gauge

$$
e^{a}{ }_{\mu}=\left(\begin{array}{cc}
N & 0 \\
N^{i} & \tilde{e}_{\beta}^{i}
\end{array}\right)
$$

often referred to as the time gauge, and this will be used in the following.

The timelike unit 1-form $n=-e^{0}$ vanishes on $\Sigma_{t}$ and the future-pointing unit vector normal to $\Sigma_{t}$ is

$$
\vec{n}=\frac{1}{N}\left(\partial_{t}-N^{\alpha} \partial_{\alpha}\right)
$$

In the time gauge (28) the extrinsic curvature of $\Sigma_{t}$ in $\mathcal{M}$ takes the form

$$
\kappa_{a b}=\left(\begin{array}{cc}
0 & 0 \\
0 & \kappa_{i j}
\end{array}\right) .
$$

$\kappa_{i j}$ can be expressed in terms of the time evolution of the dreibein

$$
\tau_{i j}:=\left(\partial_{t} \widetilde{e}_{i \alpha}\right)\left(\widetilde{e}^{-1}\right)^{\alpha}{ }_{j}
$$

and the shear of the shift function

$$
\sigma_{\{i j\}}:=\frac{1}{2}\left(\widetilde{D}_{i} N_{j}+\widetilde{D}_{j} N_{i}\right),
$$

with $\widetilde{D}_{i}$ the three-dimensional covariant derivative on $\Sigma_{t}$,

$$
\widetilde{D}_{i} N_{j}=\partial_{i} N_{j}+\widetilde{\omega}_{j k, i} N^{k} .
$$

In terms of these the extrinsic curvature is

$$
\kappa_{i j}=\frac{1}{N}\left(\tau_{\{i j\}}-\sigma_{\{i j\}}\right),
$$

with $\tau_{\{i j\}}=\frac{1}{2}\left(\tau_{i j}+\tau_{j i}\right)$.

In the time gauge the Einstein Lagrangian takes the well-known form

\footnotetext{
${ }^{8}$ Greek indices near the middle of the alphabet, $\mu, \nu, \ldots=0,1, \ldots, n$, label coordinates on $\mathcal{M}$ while indices $\alpha, \beta, \ldots=1, \ldots, n$, near the beginning of the alphabet label coordinates on $\Sigma_{t}$. Roman letters $a, b, . .=0,1, \ldots, n$ are orthonormal indices on $\mathcal{M}$ and $i, j, \ldots=1, \ldots, n$ are orthonormal indices for 1-forms $\widetilde{e}^{i}$ on $\Sigma_{t}$.
} 


$$
\begin{aligned}
L & =\frac{1}{16 \pi}\left(R_{a b} \wedge * e^{a b}-2 \Lambda * 1\right) \\
& =\frac{1}{16 \pi}\left(\kappa_{i j} \kappa^{i j}-\kappa^{2}+\widetilde{\mathcal{R}}-2 \Lambda\right) * 1 \bmod d,
\end{aligned}
$$

with $\kappa$ the trace of $\kappa_{i j}$ and $\widetilde{\mathcal{R}}$ is the three-dimensional Ricci scalar associated with $\widetilde{e}^{i}=\widetilde{e}_{\alpha}^{i} d x^{\alpha}$. Discarding surface terms $\dot{\tilde{e}}_{\alpha}^{i}$ only appears here in $\kappa_{i j}$, through the $\tau_{\{i j\}}$ term in (31), and

$$
\frac{\delta \kappa^{i j}}{\delta \dot{\widetilde{e}}_{\alpha}^{k}}=\frac{1}{N} \frac{\delta \tau^{\{i j\}}}{\dot{\widetilde{e}}_{a}^{k}}=\frac{1}{2 N}\left\{\left(\widetilde{e}^{-1}\right)^{\alpha j} \delta_{k}^{i}+\left(\widetilde{e}^{-1}\right)^{\alpha i} \delta_{k}^{j}\right\} .
$$

The momentum canonically conjugate to $\widetilde{e}_{\alpha}^{i}$ in the Hamilton formulation is the $(n+1)$-form

$$
\widetilde{\Pi}_{i}^{\alpha}=\frac{\delta L}{\dot{\delta \dot{e}^{i}}}
$$

in terms of which

$$
\begin{aligned}
\widetilde{\Pi}_{i j}:=\widetilde{e}_{\alpha}^{i} \widetilde{\Pi}^{\alpha}{ }_{i} & =\frac{1}{8 \pi N}\left(\kappa_{i j}-\delta_{i j} \kappa\right) * 1 \\
& =\frac{1}{8 \pi}\left(\kappa_{i j}-\delta_{i j} \kappa\right)(d t \wedge \widetilde{* 1}),
\end{aligned}
$$

where $\widetilde{*}$ is the Hodge-* on $\Sigma_{t}$, with $\widetilde{*} 1=\widetilde{e}^{1 \cdots n}$. We therefore define momentum $n$-forms on $\Sigma_{t}$

$$
\widetilde{\Pi}^{\alpha}{ }_{j}=\left(\widetilde{e}^{-1}\right)^{\alpha j} \widetilde{\Pi}_{i j} \quad \text { with } \quad \widetilde{\Pi}_{i j}=\frac{1}{8 \pi}\left(\kappa_{i j}-\delta_{i j} \kappa\right) \widetilde{*} .
$$

In the time gauge variations of the metric induce

$$
\boldsymbol{\Delta}_{b}^{a}=\left(\begin{array}{cc}
\frac{\delta N}{N} & 0 \\
\boldsymbol{\Delta}^{i} & \boldsymbol{\Delta}_{j}^{i}
\end{array}\right)
$$

with

$$
\boldsymbol{\Delta}^{i}=\boldsymbol{\Delta}^{i}{ }_{0}=\frac{1}{N}\left(\boldsymbol{\delta} N^{i}-\boldsymbol{\Delta}^{i}{ }_{j} N^{j}\right) \text { and } \boldsymbol{\Delta}_{j}^{i}=\left(\boldsymbol{\delta} \widetilde{e}_{\alpha}^{i}\right)\left(\widetilde{e}^{-1}\right)^{\alpha}{ }_{j} .
$$

The symplectic structure associated with the action (4) was evaluated in [3] for $\Lambda=0$, and the result is the same for nonzero $\Lambda . \boldsymbol{\theta}$ for the action (4) is

$\boldsymbol{\theta}=\frac{1}{16 \pi}\left\{\boldsymbol{\delta}\left(\omega_{a b} \wedge * e^{a b}\right)-\omega_{a b} \wedge \boldsymbol{\delta}\left(* e^{a b}\right)+d *\left(e^{a} \wedge \boldsymbol{\delta} e_{a}\right)\right\}$

and, using the expressions for $\omega_{a b}$ in the time-gauge given in Appendix B 1, Eq. (B18), this gives

$$
\Theta=-\int_{\Sigma_{t}} \widetilde{\Pi}_{i}^{\alpha} \delta^{i}{ }^{i}{ }_{\alpha}-\frac{1}{8 \pi} \boldsymbol{\delta}\left(\int_{\Sigma_{t}} \kappa * e^{0}\right)-\frac{1}{16 \pi} \int_{\partial \Sigma_{t}} \boldsymbol{\Delta}_{i} * e^{0 i},
$$

where $\widetilde{\Pi}_{i}^{\alpha} \boldsymbol{\delta}_{e^{i}}{ }_{\alpha}=\widetilde{\Pi}_{i j} S^{i j}$. In terms of the extrinsic curvature of $\Sigma_{t}$ this is
$\Theta=-\frac{1}{8 \pi} \int_{\Sigma_{t}}\left(\kappa_{i j} \boldsymbol{S}^{i j}+\boldsymbol{\delta} \kappa\right) * e^{0}-\frac{1}{16 \pi} \int_{\partial \Sigma_{t}} \boldsymbol{\Delta}_{i} * e^{0 i}$.

From (34) the presymplectic form is

$$
\boldsymbol{\Omega}=\int_{\Sigma_{t}} \boldsymbol{\delta} \widetilde{e}_{\alpha}^{i} \bar{\Lambda} \delta \widetilde{\Pi}^{\alpha}{ }_{i}-\frac{1}{16 \pi} \boldsymbol{\delta}\left(\int_{\partial \Sigma_{t}} \boldsymbol{\Delta}_{i} * e^{0 i}\right) .
$$

When the surface term vanishes this is of the Darboux form [3], the inclusion of a cosmological constant does not change this conclusion.

\section{TIMELIKE KILLING VECTORS AND MASS}

The Noether charge associated the symmetry of a timelike Killing vector is of course a mass. Suppose an asymptotically flat space-time $\mathcal{M}$ is endowed with a timelike Killing vector $\vec{K}=\frac{\partial}{\partial t}$, with the normalization fixed by demanding that $\vec{K}$ has unit length asymptotically. In the time-gauge (29) $\vec{K}$ has components

$$
K^{a}=\left(N, N^{i}\right) .
$$

It was shown in [6] that $\boldsymbol{\Phi}\left[\frac{\partial}{\partial t}\right]$ in (25) is the variation of the ADM mass. With $\partial \Sigma=\partial \Sigma_{1} \cup \partial \Sigma_{2}$

$$
\boldsymbol{\Phi}[\overrightarrow{\boldsymbol{K}}]=-\frac{1}{16 \pi} \int_{\partial \Sigma_{p}} \boldsymbol{\delta} * d \boldsymbol{K}+\int_{\partial \Sigma_{p}} i_{\overrightarrow{\boldsymbol{K}}} \boldsymbol{\theta}\left(e^{a}, \boldsymbol{\delta} e^{a}\right)
$$

is independent of $p=1$ or $p=2$.

We shall now drop the boldface notation for forms on $\mathcal{S}$ from here on-while it can be useful in understanding the general structure it becomes rather ugly when examining the details of specific examples-and write

$$
\Phi[\vec{K}]=\frac{1}{16 \pi} \int_{\partial \Sigma_{p}} \delta * d K+\int_{\partial \Sigma_{p}} i_{\vec{K}} \theta\left(e^{a}, \delta e^{a}\right) .
$$

The change in sign in the first term here is because $\delta e^{a}$ and $\overrightarrow{\boldsymbol{K}}$ anticommute in (37) while $\delta e^{a}$ and $K^{a}$ in (38) are ordinary commuting quantities.

An exact expression for $\Phi[\vec{K}]$ in the time-gauge, when $\vec{K}=\frac{\partial}{\partial t}$ in (36), is derived in Appendix B 2, Eq. (B30),

$$
\begin{aligned}
\Phi\left[\frac{\partial}{\partial t}\right]= & \frac{1}{8 \pi} \int_{\sigma}\left\{N^{2}\left(\widetilde{D}_{j}\left(\frac{S_{i}{ }^{j}}{N}\right)-\partial_{i}\left(\frac{S}{N}\right)\right)+N^{j} X_{i j}\right. \\
& \left.-N_{i}\left(S^{j k} \kappa_{j k}+\delta \kappa\right)\right\} * e^{0 i},
\end{aligned}
$$

where $S_{i j}=\frac{1}{2}\left(\Delta_{i j}+\Delta_{j i}\right), S=S^{i}{ }_{i}$ is the trace of $S_{i j}$, $\delta \kappa=\delta \kappa^{i}{ }_{i}$ is the trace of $\kappa_{i j}$ and $^{9}$

$$
\begin{aligned}
& { }^{9} \text { The combination } \\
& \qquad \delta \kappa_{i j}+\kappa_{i k} \Delta^{k}{ }_{j}-\Delta_{i k} \kappa_{j}^{k}
\end{aligned}
$$

is gauge invariant and depends only on $S_{i j}$. 


$$
X_{i j}=\delta \kappa_{i j}+\kappa_{i k} \Delta^{k}{ }_{j}-\Delta_{i k} \kappa_{j}^{k}+\kappa_{i j} S .
$$

$\sigma$ in (39) is either of the components of $\partial \Sigma, \partial \Sigma_{1}$ or $\partial \Sigma_{2}$.

\section{A. Asymptotically flat black holes}

The simplest example of the formalism in the previous section is as always the Schwarzschild line element

$d s^{2}=-\left(1-\frac{2 m}{r}\right) d t^{2}+\frac{1}{\left(1-\frac{2 m}{r}\right)} d r^{2}+r^{2}\left(d \vartheta^{2}+\sin \vartheta^{2} d \varphi^{2}\right)$

for which $N^{i}=0$. Hypersurfaces of the Schwarzschild geometry with constant $t$ are timelike for $r<2 m$ so in defining $\Sigma_{t}$ we restrict to $r>2 m$.

\section{ADM mass}

For the Schwarzschild geometry in the time-gauge $\kappa_{i j}=0$ and $N^{i}=0$ so $e^{i}=\widetilde{e}^{i}$ and Eq. (39) simplifies to

$$
\begin{aligned}
\Phi\left[\frac{\partial}{\partial t}\right]= & \frac{1}{8 \pi} \int_{\sigma}\left\{N\left(\widetilde{D}_{j}\left(S^{j}{ }_{i}\right)-\partial_{i} S\right)\right. \\
& \left.+\left(\partial_{i} N\right) S-\left(\partial_{j} N\right) S^{j}{ }_{i}\right) * e^{0 i}
\end{aligned}
$$

with $N=\sqrt{1-\frac{2 m}{r}}$.

We can choose

$$
e^{1}=\frac{d r}{\sqrt{1-\frac{2 m}{r}}}, \quad e^{2}=r d \vartheta, \quad e^{3}=r \sin \vartheta d \varphi
$$

with unit normal to $\Sigma_{t}$

$$
n=-e^{0}=-\sqrt{\left(1-\frac{2 m}{r}\right)} d t .
$$

The connection 1-forms in this gauge are

$$
\begin{array}{ll}
\omega_{01}=-\frac{m}{N r^{2}} e^{0}, & \omega_{12}=-\frac{N}{r} e^{2}, \\
\omega_{13}=-\frac{N}{r} e^{3}, & \omega_{23}=\frac{\cot \vartheta}{r} e^{2} .
\end{array}
$$

If we vary the metric by varying the mass $m \rightarrow m+\delta m$ then

$$
\delta e^{1}=\frac{\delta m}{r-2 m} e^{1}, \quad \delta e^{2}=\delta e^{3}=0,
$$

so

$$
\Delta_{j}^{i}=S_{j}^{i}=\left(\begin{array}{ccc}
\frac{\delta m}{r-2 m} & 0 & 0 \\
0 & 0 & 0 \\
0 & 0 & 0
\end{array}\right)
$$

and

$$
\delta \kappa_{i j}=0 .
$$

Now $^{10}$

$$
\widetilde{D}_{j} S_{i}{ }^{j}-\partial_{i} S_{j}{ }^{j}=\frac{2 \delta m}{r^{2}\left(1-\frac{2 m}{r}\right)^{\frac{1}{2}}} \delta_{i}^{1} \text { and } \partial_{i} N=\frac{m}{r^{2}\left(1-\frac{2 m}{r}\right)^{\frac{1}{2}}} \delta_{i}^{1},
$$

giving (with $* 1=e^{0123}$ and $* e^{01}=e^{23}$ )

$\Phi\left[\frac{\partial}{\partial t}\right]=\frac{1}{8 \pi} \int_{\sigma}\left(\frac{2 \delta m}{r^{2}}\right) r^{2} \sin \vartheta d \vartheta d \varphi=\frac{\delta m}{4 \pi} \int_{\sigma} \sin \vartheta d \vartheta d \varphi$.

For example we could take $\Sigma$ to be a thick solid shell with $r_{1} \leq r \leq r_{2}$, then the boundary $\partial \Sigma$ consists of two spheres with radii $r_{1}$ and $r_{2}$ (in particular it is not necessary to take $\left.r_{2} \rightarrow \infty\right)$. Taking $\sigma$ to be the outer sphere gives

$$
\delta \mathcal{Q}=\Phi=\frac{\delta m}{4 \pi} \int_{S^{2}} \sin \vartheta d \vartheta d \varphi=\delta m
$$

so $\Phi$ is the variation of the mass parameter, which is therefore identified with the physical mass. Since $\Phi\left[\frac{\partial}{\partial t}\right]$ is independent of $r$ we can calculate it using whatever value of $r$ is convenient. Indeed we can even smoothly distort $\sigma$ to any arbitrary shape, as long as it encloses the origin and subtends a solid angle of $4 \pi$ we will always get the same answer. ${ }^{11}$ More generally, for any space-time with a stationary metric which is asymptotically flat, we can evaluate $\Phi\left[\frac{\partial}{\partial t}\right]$ on a sphere of large $r$ in polar coordinates. Since $\frac{\partial}{\partial t}$ is Killing $\tau_{\{i j\}}$ vanishes in (31), and we can assume that

$$
\begin{aligned}
& N \sim 1+O\left(\frac{1}{r}\right), \quad N_{i} \sim O\left(\frac{1}{r}\right), \\
& \partial_{i} N \sim \widetilde{D}_{i} N_{j} \sim \kappa_{i j} \sim O\left(\frac{1}{r^{2}}\right) .
\end{aligned}
$$

These conditions include the case of asymptotically flat rotating black holes. A stationary metric has $\frac{\partial}{\partial t}$ as a Killing vector but we do not assume that the variation $\delta e^{a}$ shares this symmetry, we can only assume the falloff

$$
S_{j}^{i} \sim O\left(\frac{1}{r}\right) \quad \widetilde{D}_{i} S^{j}{ }_{k} \sim O\left(\frac{1}{r^{2}}\right) \quad \delta \kappa_{\{i j\}} \sim O\left(\frac{1}{r}\right),
$$

the last since $\delta \tau_{\{i j\}}$ could be of order $1 / r$ in (31). Here however the linearized equations of motion are invoked and satisfying these requires that, for an asymptotically flat metric,

\footnotetext{
${ }^{10}$ In an orthonormal basis $\partial_{i}:=\left(\widetilde{e}^{-1}\right)^{\alpha}{ }_{i} \partial_{\alpha}$.

${ }^{11}$ Of course the value that we get for the mass depends on the normalization of the Killing vector and, for asymptotically flat space-times, this is naturally fixed by demanding that $\vec{K}$ has unit length when $r \rightarrow \infty$.
} 


$$
\delta \tau_{\{i j\}} \sim O\left(\frac{1}{r^{2}}\right) .
$$

With these asymptotic conditions, $N^{j} X_{i j} \sim O\left(\frac{1}{r^{3}}\right)$ and (39) reduces to

$$
\Phi\left[\frac{\partial}{\partial t}\right]=\frac{1}{8 \pi} \int_{S^{2}}\left(\widetilde{D}_{j}\left(S_{i}{ }^{j}\right)-\partial_{i} S\right) \widetilde{*} \widetilde{e}^{i}+O\left(\frac{1}{r}\right),
$$

which is the variation of the ADM mass [6,9].

This variation has been calculated here asymptotically as $r \rightarrow \infty$ but it is stressed that, in principle at least, this is not necessary — we can smoothly distort the sphere at infinity to any other sphere [provided we do not pass through any matter to reach it by the distortion, otherwise (4) is not the correct action to use], and the formalism ensures that we would have obtained the same answer for any stationary asymptotically flat metric. It is not necessary to go to asymptotia to evaluate variations in the ADM mass for a stationary space-time.

\section{The Brown-York mass}

It is well-known that in asymptotically flat space-time the ADM mass [9] is related to the Brown-York mass [16], indeed it is usually stated that they are exactly equivalent. The earliest reference to their equivalence appears to be [19]. In this section we shall investigate how this relates to the Wald formalism of Sec. IVA, specifically Eq. (43).

The Brown-York mass in asymptotically flat space-time is defined using the extrinsic curvature of the asymptotic boundary of $\Sigma$. If $\widetilde{n}$ is the unit normal to $\partial \Sigma$ then the extrinsic curvature of $\partial \Sigma$ in $\Sigma$ is

$$
\widetilde{\kappa}_{i j}=\frac{1}{2} \widetilde{P}_{i}^{k} \widetilde{P}_{j}^{l}\left(\widetilde{D}_{k} \widetilde{n}_{l}+\widetilde{D}_{l} \widetilde{n}_{k}\right),
$$

where

$$
\widetilde{P}_{i}^{j}=\left(\delta_{i}^{j}-\widetilde{n}_{i} \widetilde{n}^{j}\right)
$$

projects from the (co)tangent space of $\Sigma$ onto the (co) tangent space of $\partial \Sigma$. The trace $\widetilde{\kappa}=\widetilde{\kappa}_{i}{ }^{i}$ can be obtained from

$$
\widetilde{d} \widetilde{*}=\widetilde{\kappa} \widetilde{*} 1,
$$

where $\widetilde{d}=\widetilde{e}^{i} \partial_{i}$ is the exterior derivative and $\widetilde{*}$ the Hodge duality operator on $\Sigma_{t}$ at constant $t$. If $\widetilde{\kappa}_{0}$ is the trace of the extrinsic curvature of $\partial \Sigma$ with the flat metric on $\Sigma$ then the Brown-York mass [16] is

$$
M_{B-Y}=\frac{1}{8 \pi} \int_{\left.S^{2}\right|_{t, \infty}}\left(\widetilde{\kappa}_{0}-\widetilde{\kappa}\right) \widetilde{*} \widetilde{n}=-\frac{1}{8 \pi} \int_{\left.S^{2}\right|_{t, \infty}}(\delta \widetilde{\kappa}) \widetilde{*} \widetilde{n} .
$$

This is related to (43) as follows. Under a perturbation of the metric (44) can be used to show that, in the gauge (28),

$$
\int_{\sigma}\left\{(\delta \widetilde{\kappa})+\widetilde{\kappa}_{i j} S^{i j}\right\} \widetilde{*} \widetilde{n}=\int_{\sigma}\left(\partial_{i} S-\widetilde{D}_{j} S^{j}{ }_{i}\right) \widetilde{*} \widetilde{e}^{i} .
$$

Hence, at large $r$ in asymptotically flat space-time with the falloff conditions (42),

$$
\Phi\left[\frac{\partial}{\partial t}\right]=-\frac{1}{8 \pi} \int_{\left.S^{2}\right|_{t, r}}\left\{(\delta \widetilde{\kappa})+\widetilde{\kappa}_{i j} S^{i j}\right\} \widetilde{*} \widetilde{n}+O\left(\frac{1}{r}\right) .
$$

Now let $\delta \widetilde{\kappa}=\widetilde{\kappa}-\widetilde{\kappa}_{0}$ be the deviation of the trace of the extrinsic curvature of the asymptotic boundary $S^{2}$ of $\Sigma$ from its flat space value

$$
\widetilde{\kappa}_{0}=\frac{2}{r} .
$$

Asymptotically the extrinsic curvature has the form

$$
\widetilde{\kappa}_{i j}=\frac{1}{r} \widetilde{P}_{i j}+O\left(\frac{1}{r^{2}}\right) .
$$

This implies that $\widetilde{\kappa}_{0}-\widetilde{\kappa} \sim O\left(\frac{1}{r^{2}}\right)$, and it is these $1 / r^{2}$ terms that contain information about the mass since

$$
\frac{1}{r^{2}} \int_{\left.S^{2}\right|_{t, r}} \widetilde{*} \widetilde{n}=4 \pi+O\left(\frac{1}{r}\right)
$$

and

$\Phi\left[\frac{\partial}{\partial t}\right]=\frac{1}{8 \pi} \int_{\left.S^{2}\right|_{t, \infty}}\left(\widetilde{\kappa}_{0}-\widetilde{\kappa}\right) \widetilde{*} \widetilde{n}+\lim _{r \rightarrow \infty}\left(\frac{1}{8 \pi r} \int_{\left.S^{2}\right|_{t, r}} S_{\perp} \widetilde{*} \tilde{n}\right)$,

where $S_{\perp}=\widetilde{P}_{i j} S^{i j}$ is the transverse trace of $S_{i j}$ [it does not matter whether or not we use the flat metric for $\widetilde{*} \widetilde{n}$ in (47) since the difference is $\left.O\left(\frac{1}{r}\right)\right]$. We see from (45) that $\Phi$ equals the Brown-York mass if

$$
\lim _{r \rightarrow \infty}\left(\frac{1}{r} \int_{\left.S^{2}\right|_{t, r}} S_{\perp} \widetilde{*} \widetilde{n}\right)=0 .
$$

It is not sufficient that $S_{i j}$ falls off like $1 / r$, in addition the transverse trace of the metric perturbation, $S_{\perp}$, must fall off faster than $1 / r$ (for a perturbation corresponding to a gravitational wave moving radially outward this is guaranteed since $S_{\perp}=0$ ).

This analysis shows that the difference $\widetilde{\kappa}_{0}-\widetilde{\kappa}$ in the definition of the Brown-York mass is best viewed as a 1 -form on the space of solutions $\mathcal{S}$.

\section{The Bondi mass}

In Bondi-Sachs coordinates $(u, r, \vartheta, \varphi),[21]$, the line element is

$$
\begin{aligned}
d^{2} s= & -U^{2} e^{2 W} d u^{2}-2 e^{2 W} d u d r \\
& +r^{2} h_{\alpha \beta}\left(d x^{\alpha}-V^{\alpha} d u\right)\left(d x^{\beta}-V^{\beta} d u\right),
\end{aligned}
$$

where $u=t-r$ is a lightlike coordinate, $r$ is a radial coordinate and $x^{\alpha}=(\vartheta, \varphi)$ are coordinates on a 2 -sphere 
with metric components $h_{\alpha \beta}$. In general $U(u, r, \vartheta, \varphi)$, $W(u, r, \vartheta, \varphi)$ and $V^{\alpha}(u, r, \vartheta, \varphi)$ are functions of all four coordinates, but we require at least that

$$
U \underset{r \rightarrow \infty}{\longrightarrow} 1, \quad W \underset{r \rightarrow \infty}{\longrightarrow} 1, \quad V^{\alpha} \underset{r \rightarrow \infty}{\longrightarrow} 0 .
$$

With foresight in relation to the Bondi mass it is useful to replace $U$, without any loss of generality, with the function $M(u, r, \vartheta, \varphi)$ defined via

$$
U=1-\frac{2 M}{r}
$$

with $M$ finite as $r \rightarrow \infty$.

We can choose orthonormal 1-forms

$$
\begin{aligned}
& e^{0}=\exp (W)\left(U d u+U^{-1} d r\right), \\
& e^{1}=U^{-1} \exp (W) d r, \quad e^{i}=r \widetilde{e}^{i}-V^{i} d u,
\end{aligned}
$$

with $\widetilde{e}^{i}(i=2,3)$ orthonormal 1-forms for the 2-sphere metric $h_{\alpha \beta}$ and

$$
V^{i}=r \widetilde{e}_{\alpha}^{i} V^{\alpha}
$$

We are free to choose a gauge in which

$$
\widetilde{e}^{i}=C_{j}^{i} \hat{e}^{i},
$$

where $\operatorname{det} C=1$ and

$$
\hat{e}^{2}=d \vartheta, \quad \hat{e}^{3}=\sin \vartheta d \varphi
$$

are orthonormal 1-forms for the round unit 2-sphere. Asymptotically we demand that $C^{i}{ }_{j}=\delta^{i}{ }_{j}+O\left(\frac{1}{r}\right)$ for large $r$ but the condition $\operatorname{det} C=1$ ensures that volume of the 2 -sphere is $4 \pi$ for all $r$ (see Appendix C). We shall call (50) and (51) the Bondi-Sachs gauge.

Now $\vec{K}=\frac{\partial}{\partial t}=\frac{\partial}{\partial u}$ has metric dual 1-form

$$
K=-U \exp (W) e^{0}-V_{i} e^{i}
$$

and using this one finds that, on a sphere defined by $u$ and $r$ constant,

$$
\begin{aligned}
\frac{1}{16 \pi} \int_{S^{2}} \delta(* d K)= & \frac{1}{8 \pi} \delta\left(\int _ { S ^ { 2 } } \left\{\frac{1}{2}\left(U^{2}\right)^{\prime}+U^{2} W^{\prime}-\dot{W}\right.\right. \\
& \left.\left.-V^{i} \partial_{i} W-\frac{1}{2} e^{-2 W} V^{i} V_{i}^{\prime}\right\} r^{2} \hat{e}^{23}\right),
\end{aligned}
$$

with $\dot{W}=\partial_{u} W$ and $W^{\prime}=\partial_{r} W$. The expression for $\int_{S^{2}} i_{\vec{K}} \theta$ is more complicated but if we assume that $V^{i} \sim \frac{1}{r}$ and $W=1+O\left(\frac{1}{r}\right)$, in order to ensure asymptotic flatness, it takes the asymptotic form

$$
\begin{aligned}
\int_{S^{2}} i_{\vec{K}} \theta= & \frac{1}{8 \pi} \int_{S^{2}}\left\{\frac{\delta M}{r^{2}}+\delta \dot{W}-\delta W^{\prime}\right. \\
& \left.+\left(1-\frac{2 M}{r}\right) \frac{\delta W}{r}\right\} r^{2} \hat{e}^{23}+O\left(\frac{1}{r}\right) .
\end{aligned}
$$

Adding (52) and (53) the $\delta \dot{W}$ terms cancel and

$$
\begin{aligned}
& \frac{1}{16 \pi}\left(\int_{S^{2}} \delta(* d K)\right)+\int_{S^{2}} i_{\vec{K}} \theta \\
& =\frac{1}{8 \pi} \int_{S^{2}}\{2(\delta M-M \delta W) \\
& \left.\quad+r\left[\delta W-\delta M^{\prime}-2 \delta\left(M W^{\prime}\right)\right]-r^{2} \delta W^{\prime}\right\} \hat{e}^{23}+O\left(\frac{1}{r}\right) .
\end{aligned}
$$

Demanding that the metric is asymptotically flat imposes the conditions

$$
\begin{aligned}
& M(u, r, \vartheta, \varphi)=m(u, \vartheta, \varphi)+O\left(\frac{1}{r}\right), \\
& W(u, r, \vartheta, \varphi)=\frac{w(u, \vartheta, \varphi)}{r}+O\left(\frac{1}{r^{2}}\right),
\end{aligned}
$$

giving

$$
\begin{aligned}
& \frac{1}{16 \pi}\left(\int_{S^{2}} \delta(* d K)\right)+\int_{S^{2}} i_{\vec{K}} \theta \\
& \quad=\frac{1}{4 \pi} \int_{S^{2}}(\delta m+\delta w) \hat{e}^{23}+O\left(\frac{1}{r}\right) .
\end{aligned}
$$

In general the Bondi mass is defined to be

$$
M(u)=\frac{1}{4 \pi} \int_{S^{2}} m(u, \vartheta, \varphi) \sin \vartheta d \vartheta d \varphi,
$$

and here we invoke the linearized equations of motion, at order $\frac{1}{r^{3}}$ the Einstein equations actually require that $W \sim \frac{1}{r^{2}}$ so $\delta W \sim \frac{1}{r^{2}}$ also and $\delta w=0$.

Finally

$$
\frac{1}{16 \pi}\left(\int_{S^{2}} \delta(* d K)\right)+\int_{S^{2}} i_{\vec{K}} \theta=\delta M+O\left(\frac{1}{r}\right),
$$

so Wald's expression indeed equals the variation of the Bondi mass. Again, since $\vec{K}$ is killing, the general formalism ensures that any value of $r$ could have been used in the calculating the left-hand side of (57) and the answer would always be the same.

\section{CONCLUSIONS}

The phase space formulation of a dynamical system is ideally suited to the discussion of conserved quantities and symmetries of relativistic systems which are invariant under diffeomorphisms, such as general relativity, are no exception. For general relativity the symplectic form $\boldsymbol{\Omega}$ was 
derived by Witten and Crnković in [1] and reformulated by Lee and Wald where it was shown in [3] to have the Darboux form in asymptotically flat space-times. This conclusion is not changed when a cosmological constant is included.

For stationary solutions of Einstein's equations, when $\frac{\partial}{\partial t}$ is Killing, $\Phi$ in (38) is a 2-form on the space of solutions which is independent of the surface [more generally $(n-1)$-dimensional submanifold] $\sigma$ in $\mathcal{M}$ on which it is calculated.

If the solution is that of an asymptotically flat stationary space-time $\Phi$ is the variation of both the ADM mass and the Brown-York mass, when $\Sigma$ is spacelike and yields the Bondi mass when $\Sigma$ is an appropriately chosen lightlike hypersurface respectively. The analysis here lends further support to the suggestion that Lee and Wald's expression $\Phi\left[e^{a}, \mathcal{L}_{\frac{\partial}{\partial t}} e^{a}, \delta e^{a}\right]$ in Eq. (38) thus serves to unify the different definitions of mass in general relativity that appear in the literature and is a universal expression for the variation of the mass associated with a stationary solution of any diffeomorphism invariant theory.

From a mathematical point of view the construction fits very neatly into a double complex that captures the cohomology of the various forms involved, details of this mathematical structure are given elsewhere [8].

\section{ACKNOWLEDGMENTS}

The author acknowledges support by the Action MP1405 QSPACE from the European Cooperation in Science and Technology (COST).

\section{APPENDIX A: DIFFERENTIAL FORM NOTATION}

\section{Connection and curvature}

For a given metric let $e^{a}$ denote a set of associated orthonormal 1-forms (a tetrad in four dimensions). Our conventions are that orthonormal indices are raised and lowered with

$$
\eta_{a b}=\eta^{a b}=\left(\begin{array}{cccc}
-1 & 0 & 0 & 0 \\
0 & 1 & 0 & 0 \\
0 & 0 & 1 & 0 \\
0 & 0 & 0 & 1
\end{array}\right)
$$

When 1-forms are wedged together we use the short-hand notation

$$
e^{a_{1} a_{2} \cdots a_{n}}=e^{a_{1}} \wedge e^{a_{1}} \wedge \cdots \wedge e^{a_{n}} .
$$

$i_{a}$ denotes contraction with the orthonormal vector metric dual to $e^{a}$ so, e.g.,

$$
i_{a} e^{b c}=\delta_{a}^{b} e^{c}-\delta_{a}^{c} e^{b} .
$$

The associated torsion free connection 1-forms can be expressed in terms of the $e^{a}$ as

$$
\omega_{a b}=\frac{1}{2}\left(e^{c} i_{a} i_{b} d e_{c}-i_{a} d e_{b}+i_{b} d e_{a}\right),
$$

where $d$ is the exterior derivative. The covariant exterior derivative is denoted $D$, in terms of which the torsion free condition is

$$
D e^{a}=d e^{a}+\omega_{b}^{a} \wedge e^{b}=0 .
$$

The curvature 2-forms are

$$
R_{a b}=d \omega_{a b}+\omega_{a}^{c} \wedge \omega_{c b}=\frac{1}{2} R_{a b c d} e^{c d},
$$

where $R_{a b c d}$ are the components of the Riemann tensor in the chosen orthonormal basis. The components of the associated Ricci tensor, $\mathcal{R}_{a b}$, and the Einstein tensor, $G_{a b}$, can be extracted from

$$
R_{b c} \wedge * e^{a b c}=\left(2 \mathcal{R}^{a}{ }_{b}-\mathcal{R} \delta^{a}{ }_{b}\right) * e^{b}=-2 G^{a}{ }_{b} * e^{b},
$$

where $\mathcal{R}=\mathcal{R}^{a}{ }_{a}$ is the Ricci scalar and $*$ is the Hodge duality operator.

If the metric is varied infinitesimally the orthonormal 1 -forms must change,

$$
e^{a} \rightarrow e^{a}+\delta e^{a} .
$$

Demanding that the connection 1-forms also change so as to preserve the torsion free condition implies that

$$
\delta\left(D e^{a}\right)=D \delta e^{a}+\delta\left(\omega_{b}^{a}\right) \wedge e^{b}=0
$$

allowing $\delta \omega_{a b}$ to be determined from $\omega_{a b}$ and $\delta e^{a}$ through

$$
\delta \omega_{a b}=\frac{1}{2}\left(e^{c} i_{a} i_{b} D \delta e_{c}-i_{a} D \delta e_{b}+i_{b} D \delta e_{a}\right) .
$$

The variation in the curvature 2 -forms is

$\delta R_{a b}=d\left(\delta \omega_{a b}\right)+\omega_{a}^{c} \wedge \delta \omega_{c b}+\omega_{b}{ }^{c} \wedge \delta \omega_{a c}=D(\delta \omega)_{a b}$.

\section{APPENDIX B: EXPLICIT EXPRESSION FOR $\theta$ AND $\omega$ IN EINSTEIN GRAVITY}

From (A3), keeping only symmetric variations,

$$
\theta\left(e^{a}, \delta e^{a}\right)=\frac{1}{8 \pi}\left(D_{b} S_{a}^{b}-\partial_{a} S_{b}^{b}\right) * e^{a} .
$$

For completeness we give here the explicit form of $\omega$ under two variations, $\delta_{1}$ and $\delta_{2}$ with 


$$
\begin{aligned}
\left(S_{1}\right)^{a}{ }_{b} & =\left(\delta_{1} \widetilde{e}^{a}{ }_{\mu}\right)\left(\widetilde{e}^{-1}\right)_{b}^{\mu} \text { and } \\
\left(S_{2}\right)^{a}{ }_{b} & =\left(\delta_{2} \widetilde{e}^{a}{ }_{\mu}\right)\left(\widetilde{e}^{-1}\right)_{b}^{\mu}, \\
\omega\left(e^{a}, \delta_{1} e^{a}, \delta_{2} e^{2}\right)= & \frac{1}{8 \pi}\left\{\left(S_{1}\right)^{b c} D_{a}\left(S_{2}\right)_{b c}-2\left(S_{1}\right)^{b c} D_{c}\left(S_{2}\right)_{b a}\right. \\
& \left.+S_{1} D_{b}\left(S_{2}\right)_{a}^{b}+\left(S_{1}\right)_{a}^{b} \partial_{b} S_{2}-S_{1} \partial_{a} S_{2}\right\} * e^{a} \\
& -(1 \leftrightarrow 2),
\end{aligned}
$$

where $S_{1}:=\left(S_{1}\right)_{c}{ }^{c}$ is the trace of $\left(S_{1}\right)_{b}{ }^{c}$ and similarly for $S_{2}$. The explicit form of $\omega$ is not very useful but of course it vanishes if either $\left(S_{1}\right)_{a b}$ or $\left(S_{2}\right)_{a b}$ is zero, in particular this is the case if either of the variations is generated by a Killing symmetry.

\section{Spacelike foliation}

For a space-time $\mathcal{M}$ with metric $g_{\mu \nu}$ and coordinates $x^{\mu}$ foliate $\mathcal{M}$ with constant time hypersurfaces. Let $x^{\mu}=$ $\left(t, x^{\alpha}\right)$ where $\alpha=1,2,3$ and $t$ is a time coordinate. We use the standard ADM decomposition: assume that $t=$ const are spacelike hypersurfaces, $\Sigma_{t}$, and denote the induced metric on $\Sigma_{t}$ by $h_{\alpha \beta}(t)$. The four-dimensional line element decomposes as

$$
\begin{aligned}
d s^{2} & =g_{\mu \nu} d x^{\mu} d x^{\nu}=g_{t t} d t^{2}+2 g_{t \alpha} d t d x^{\alpha}+g_{\alpha \beta} d x^{\alpha} d x^{\beta} \\
& =-N^{2} d t^{2}+h_{\alpha \beta}\left(d x^{\alpha}+N^{\alpha} d t\right)\left(d x^{\beta}+N^{\beta} d t\right),
\end{aligned}
$$

where $g_{t t}=-N^{2}+h_{\alpha \beta} N^{\alpha} N^{\beta}, g_{t \alpha}=g_{\alpha \beta} N^{\beta}$ and $h_{\alpha \beta}=g_{\alpha \beta}$.

The orthonormal 1 -forms $e^{a}$ for the metric $g$ can be expressed in a coordinate basis as

$$
e^{a}=e_{\mu}^{a} d x^{\mu}
$$

while

$$
\widetilde{e}^{i}=\widetilde{e}_{\alpha}^{i} d x^{\alpha},
$$

with $i=1,2,3$, are orthonormal 1-forms for $h$. Then $\widetilde{e}_{\alpha}^{i}=e_{\alpha}^{i}$ and

$$
e^{0}=N d t \quad \text { and } \quad e^{i}=\widetilde{e}^{i}+\frac{N^{i}}{N} e^{0}
$$

with $N^{i}=e^{i}{ }_{\alpha} N^{\alpha}$ the orthonormal components of the shift vector. The connection 1-forms on $\Sigma_{t}$ are defined in the usual way

$$
\widetilde{d} \widetilde{e}^{i}+\widetilde{\omega}_{j}^{i} \wedge \widetilde{e}^{j}=0
$$

with $\widetilde{d}=\widetilde{e}^{i} \partial_{i}$ the exterior derivative on $\Sigma_{t}$ at constant $t$.

In this gauge

$$
e^{a}{ }_{\mu}=\left(\begin{array}{cc}
N & 0 \\
N^{i} & \widetilde{e}_{\beta}^{i}
\end{array}\right), \quad\left(e^{-1}\right)^{\mu}{ }_{a}=\left(\begin{array}{cc}
\frac{1}{N} & 0 \\
-\frac{N^{\alpha}}{N} & \left(\widetilde{e}^{-1}\right)^{\alpha}{ }_{j}
\end{array}\right),
$$

and the unit vector normal to $\Sigma_{t}, \vec{n}$, has orthonormal components $n^{a}=(1,0,0,0)$ so the metric dual 1-form is $n=n_{a} e^{a}=-e^{0}$.

Metric variations are described by

$$
\Delta^{a}{ }_{b}=\left(\begin{array}{cc}
\frac{\delta N}{N} & 0 \\
{\widetilde{e^{i}}}_{\alpha} \delta N^{\alpha} & \Delta^{i}{ }_{j}
\end{array}\right),
$$

with $\Delta^{i}{ }_{j}=\left(\delta \widetilde{e}^{i}{ }_{\alpha}\right)\left(\widetilde{e}^{-1}\right)^{\alpha}{ }_{j}$. This can be decomposed into symmetric and antisymmetric parts

$$
S_{i j}=\Delta_{\{i j\}}=\frac{1}{2}\left(\Delta_{i j}+\Delta_{j i}\right), \quad A_{i j}=\Delta_{[i j]}=\frac{1}{2}\left(\Delta_{i j}-\Delta_{j i}\right) .
$$

If we define the shift 1 -forms as

$$
\widetilde{N}=h_{\alpha \beta} N^{\alpha} d x^{\beta}=N_{i} \widetilde{e}^{i}
$$

then $^{12}$

$$
\delta \widetilde{N}=(\delta \widetilde{N})_{i} e^{i} \quad \text { with } \quad(\delta \widetilde{N})_{i}=\delta N_{i}+N_{j} \Delta^{j}{ }_{i},
$$

and

$\Delta^{i}:=\Delta^{i}{ }_{0}=\frac{\widetilde{e}_{\alpha}^{i} \delta N^{\alpha}}{N}=\frac{1}{N}\left(\delta N^{i}-\Delta^{i}{ }_{j} N^{j}\right)=\frac{1}{N}\left((\delta \widetilde{N})^{i}-2 S_{j}^{i} N^{j}\right)$.

The vector $\vec{K}=\frac{\partial}{\partial t}$ has contravariant components $K^{\mu}=(1,0,0,0)$, so $K^{a}=\left(N, N^{i}\right)$ and the metric dual 1 -form is

$$
K=-N e^{0}+N_{i} e^{i}
$$

Under the diffeomorphism generated by $\frac{\partial}{\partial t}$ the change in the metric components is $\partial_{t} g_{\mu \nu}$, and we define

$$
\tau^{a}{ }_{b}=\dot{e}_{\mu}^{a}\left(e^{-1}\right)^{\mu}{ }_{b},
$$

where ${ }^{\cdot}=\partial_{t}$, so

$$
\tau_{0}^{i}=\frac{\left(\widetilde{e}_{\alpha}^{i}\right) \dot{N}^{\alpha}}{N}
$$

and

$$
\dot{\tilde{e}}^{i}=\dot{\tilde{e}}_{\alpha}^{i} d x^{\alpha}=\tau_{j}^{i} \widetilde{e}^{j},
$$

where

\footnotetext{
${ }^{12} N_{i} \widetilde{e}^{i}$ is invariant under spatial gauge transformations, $\Delta_{i j}=A_{i j},(\delta \widetilde{N})_{i}=0$, so $\delta N^{i}=-N^{k} A_{k i}$ under such a gauge transformation.
} 


$$
\tau_{j}^{i}=\dot{\tilde{e}}_{\alpha}^{i}\left(\widetilde{e}^{-1}\right)^{\alpha}{ }_{j}
$$

If $\frac{\partial}{\partial t}=\vec{K}$ is Killing then

$$
\mathcal{L}_{\vec{K}} g_{\mu \nu}=\frac{\partial g_{\mu \nu}}{\partial t}:=\dot{g}_{\mu \nu}=0
$$

in this basis, so $\dot{h}_{\alpha \beta}, \dot{N}^{\alpha}$ and $\dot{N}$ all vanish. However $\dot{N}^{i}$ is not necessarily zero, only $\dot{N}^{\alpha}=0$ and $\dot{e}_{0}^{i}=0$, and

$$
\dot{N}^{i}=\dot{e}_{\alpha}^{i} N^{\alpha}
$$

so the orthonormal triad $\widetilde{e}^{i}$ are not necessarily time independent, even if the metric $h$ is. While $\tau_{\{i j\}}$ is zero when $\frac{\partial}{\partial t}$ is Killing $\tau_{[i j]}$ need not be, rather $\dot{N}^{\alpha}=0$ so

$$
\dot{N}_{i}-\tau_{[i j]} N^{j}=0
$$

In general the connection 1-forms in the gauge (B5) are ${ }^{13}$

$$
\begin{aligned}
& \omega_{0 i}=-\frac{\partial_{i} N}{N} e^{0}+\frac{1}{N}\left(\sigma_{\{i j\}}-\tau_{\{i j\}}\right) e^{j} \\
& \omega_{i j}=\widetilde{\omega}_{i j}-\frac{1}{N}\left(\sigma_{[i j]}+\tau_{[i j]}\right) e^{0},
\end{aligned}
$$

where $\sigma_{\{i j\}}$ and $\sigma_{[i j]}$ are the shear and vorticity of the shift vector,

$$
\sigma_{\{i j\}}:=\frac{1}{2}\left(\widetilde{D}_{i} N_{j}+\widetilde{D}_{j} N_{i}\right), \quad \sigma_{[i j]}:=\frac{1}{2}\left(\widetilde{D}_{i} N_{j}-\widetilde{D}_{j} N_{i}\right),
$$

with

$$
\sigma_{i j}=\widetilde{D}_{i} N_{j}=\partial_{i} N_{j}+\widetilde{\omega}_{j k, i} N^{k}
$$

the covariant derivative of the shift functions on $\Sigma_{t}$.

The extrinsic curvature of $\Sigma_{t}$ is

$$
\kappa_{a b}=\frac{1}{2}\left(\delta_{a}{ }^{c}-n_{a} n^{c}\right)\left(\delta_{b}{ }^{d}-n_{b} n^{d}\right)\left(D_{c} n_{d}+D_{c} n_{d}\right) .
$$

In the time-gauge, $n_{a}=(-1,0,0,0)$, and

$$
D_{a} n_{b}=\partial_{a} n_{b}+n^{c} \omega_{b c, a}=-\omega_{b 0, a}
$$

so

${ }^{13}$ By definition

$$
\widetilde{\omega}_{i j}=\widetilde{\omega}_{i j, k} \widetilde{e}^{k}=\widetilde{\omega}_{i j, k}\left(e^{k}-\frac{N^{k}}{N} e^{0}\right)
$$

so

$$
\omega_{i j, 0}=-\frac{1}{N}\left(\sigma_{[i j]}+\tau_{[i j]}+N^{k} \widetilde{\omega}_{i j, k}\right) .
$$

$$
\begin{aligned}
\kappa_{a b} & =\left(\begin{array}{cc}
0 & 0 \\
0 & \kappa_{i j}
\end{array}\right) \text { with } \\
\kappa_{i j} & =\frac{1}{2}\left(\omega_{i 0, j}+\omega_{j 0, i}\right)=\frac{1}{N}\left(\tau_{\{i j\}}-\sigma_{\{i j\}}\right) .
\end{aligned}
$$

We can rewrite (B14) as

$$
\begin{gathered}
\omega_{0 i}=-\frac{\partial_{i} N}{N} e^{0}-\kappa_{i j} e^{j} \\
\omega_{i j}=\widetilde{\omega}_{i j}-\frac{1}{N}\left(\sigma_{[i j]}+\tau_{[i j]}\right) e^{0} .
\end{gathered}
$$

Under an infinitesimal variation, ${ }^{14}$ with $\Delta^{0}{ }_{i}=0$ maintaining the gauge (B5),

$$
\begin{aligned}
& \left(\delta \omega_{0 i}\right)_{0}=-\frac{\delta\left(\partial_{i} N\right)}{N}-\kappa_{i j} \Delta^{j} \\
& \left(\delta \omega_{0 i}\right)_{j}=-\delta \kappa_{i j}-\kappa_{i k} \Delta^{k}{ }_{j}
\end{aligned}
$$

$$
\begin{aligned}
\left(\delta \omega_{i j}\right)_{0} & =-\frac{1}{N}\left(\sigma_{[i j]}+\tau_{[i j]}+\left(\delta \widetilde{\omega}_{i j}\right)_{k} N^{k}\right) \\
\left(\delta \omega_{i j}\right)_{k} & =\left(\delta \widetilde{\omega}_{i j}\right)_{k} .
\end{aligned}
$$

Having performed the variation we can now set $\tau_{\{i j\}}=0$ for a stationary solution, but $\tau_{[i j]}$ in (B18) is still arbitrary, though it must always drop out of any physical quantities.

\section{Exact expression for $\Phi[\vec{K}]$}

First we collect together all the pieces we need to calculate $\Omega$

(1) Firstly

$$
\begin{aligned}
\int_{\partial \Sigma_{t}} & i_{\vec{K}}\left(\delta \omega_{a b} \wedge * e^{a b}\right) \\
= & 2 \int_{\partial \Sigma_{t}} i_{\vec{K}}\left\{\left(\delta \omega_{a}^{b}\right)_{b} * e^{a}\right\} \\
= & 2 \int_{\partial \Sigma_{t}}\left\{N\left(\delta \widetilde{\omega}_{i}^{j}\right)_{j}-\delta\left(\partial_{i} N\right)-N \kappa_{i j} \Delta^{j}\right. \\
& \left.-N_{i}\left(\kappa_{j k} S^{j k}+\delta \kappa\right)\right\} * e^{0 i},
\end{aligned}
$$

where $\delta \kappa=\delta \kappa^{i}{ }_{i}$ is the trace of the variation of the extrinsic curvature of $\Sigma_{t}$ in $\mathcal{M}$.

(2) Next we need $\int_{\partial \Sigma_{t}} i_{\vec{K}} d *\left(e^{a} \wedge \delta e_{a}\right)$. This can be evaluated using

$$
\mathcal{L}_{\vec{K}} \alpha=\partial_{t} \alpha,
$$

\footnotetext{
${ }^{14}$ Note that $\delta \omega_{a b}=\delta \omega_{a b, c} e^{c}+\omega_{a b, c} \delta e^{c}=\left(\delta \omega_{a b, c}+\omega_{a b, d} \Delta^{d}{ }_{c}\right) e^{c}$, $\Rightarrow\left(\delta \omega_{a b}\right)_{c}=\delta \omega_{a b, c}+\omega_{a b, d} \Delta^{d}{ }_{c}$.

$\delta \omega_{a b, c}$ is not a tensor while $\left(\delta \omega_{a b}\right)_{c}$ is.
} 
for any $p$-form $\alpha$, to write

$$
\begin{aligned}
\int_{\partial \Sigma_{t}} i_{\vec{K}} d *\left(e^{a} \wedge \delta e_{a}\right) & =\int_{\partial \Sigma_{t}} \mathcal{L}_{\vec{K}} *\left(e^{a} \wedge \delta e_{a}\right)=\int_{\partial \Sigma_{t}} \partial_{t}\left(e^{a} \wedge \delta e_{a}\right)=\int_{\partial \Sigma_{t}} \partial_{t}\left(\Delta_{0 i}-\Delta_{i 0}\right) * e^{0 i}=-\int_{\partial \Sigma_{t}} \partial_{t}\left(\Delta_{i} * e^{0 i}\right) \\
& =-\int_{\partial \Sigma_{t}}\left(\partial_{t} \Delta_{i}+\tau_{j}^{j} \Delta_{i}-\tau_{i}{ }^{j} \Delta_{j}\right) * e^{0 i}=-\int_{\partial \Sigma_{t}}\left(\partial_{t} \Delta_{i}-\tau_{[i j]} \Delta^{j}\right) * e^{0 i}
\end{aligned}
$$

where in the last step we have assumed that $\frac{\partial}{\partial t}$ is Killing so $\tau_{\{i j\}}=0$. Now on shell

$$
\partial_{t} \Delta_{i}=\delta \tau_{i 0}+\tau_{[i j]} \Delta^{j}
$$

when $\frac{\partial}{\partial t}$ is Killing $^{15}$ for $e^{a}$ (so $\dot{N}=0, \dot{N}^{\alpha}=0 \Rightarrow \tau_{i 0}=0$, as well as $\tau_{\{i j\}}=0$ ). In this case (B23) is simply

$$
\int_{\partial \Sigma_{t}} \mathcal{L}_{\vec{K}} *\left(e^{a} \wedge \delta e_{a}\right)=-\int_{\partial \Sigma_{t}}\left(\delta \tau_{i 0}\right) * e^{0 i}
$$

(3) The final piece we need to calculate $\Omega$ is $\delta \int * d K$. First

$$
\begin{gathered}
d K=\frac{1}{N}\left(\partial_{i} N^{2}-2 \sigma_{i j} N^{j}+\dot{N}^{i}+\tau_{j i} N^{j}\right) e^{0 i}+\sigma_{[i j]} e^{i j} \\
\Rightarrow \int_{\partial \Sigma_{t}} * d K=\int_{\partial \Sigma_{t}}\left(2 \partial_{i} N-\frac{2}{N} \sigma_{i j} N^{j}+\frac{1}{N}\left(\dot{N}_{i}+\tau_{j i} N^{j}\right)\right) * e^{0 i}=\int_{\partial \Sigma_{t}}\left(2 \partial_{i} N+2 \kappa_{i j} N^{j}+\frac{1}{N}\left(\dot{N}_{i}-\tau_{i j} N^{j}\right)\right) * e^{0 i} .
\end{gathered}
$$

When $\vec{K}$ is Killing $\tau_{0}^{i}=\frac{1}{N}\left(\dot{N}^{i}-\tau^{i}{ }_{j} N^{j}\right)=0$, but in general

$$
\delta\left(\dot{N}^{i}-N_{j} \tau^{i j}\right)=N \delta \tau_{0}^{i}+(\delta N) \tau_{0}^{i}=N \delta \tau_{0}^{i} \quad \text { on shell }
$$

does not vanish.

Therefore, on shell,

$$
\begin{aligned}
\delta\left(\int_{\partial \Sigma_{t}} * d K\right) & =\int_{\partial \Sigma_{t}}\left\{2 \delta\left(\partial_{i} N+\kappa_{i j} N^{j}\right)+2\left(\partial_{j} N+\kappa_{j k} N^{k}\right)\left(\delta_{i}^{j} S-\Delta_{i}^{j}\right)+\delta \tau_{i 0}\right\} * e^{0 i} \\
& =\int_{\partial \Sigma_{t}}\left\{2 X_{i j} N^{j}+2 N \kappa_{i j} \Delta^{j}-2 \Delta_{i}^{j} \partial_{j} N+2\left(\partial_{i} N\right) S+\delta \tau_{i 0}\right\} * e^{0 i}
\end{aligned}
$$

where

$$
X_{i j}=\delta \kappa_{i j}+[\kappa, \Delta]_{i j}+\kappa_{i j} S
$$

with $S=S^{k}{ }_{k}$ and $[\kappa, \Delta]_{i j}$ is the commutator of the matrices $\kappa_{i j}$ and $\Delta_{i j}$.

Assembling (B22), (B24) and (B25) one finds

$$
\begin{aligned}
\Omega\left[e^{a}, \mathcal{L}_{\vec{K}} e^{a}, \delta e^{a}\right] & =\frac{1}{16 \pi} \int_{\partial \Sigma_{t}}\left\{2 i_{\vec{K}}\left(\delta \omega_{a b} \wedge * e^{a b}\right) i_{\vec{K}} d *\left(e^{a} \wedge \delta e_{a}\right)+\delta * d K\right\} \\
& =\frac{1}{8 \pi} \int_{\partial \Sigma_{t}}\left\{N\left(\delta \widetilde{\omega}_{i}^{j}\right)_{j}-\left(\partial_{j} N\right) \Delta_{i}^{j}+\left(\partial_{i} N\right) S+X_{i j} N^{j}-N_{i}\left(\kappa_{j k} S^{j k}+\delta \kappa\right)\right\} * e^{0 i} .
\end{aligned}
$$

\footnotetext{
${ }^{15}$ Note that, although $\tau_{0}^{i}=0$ when $\frac{\partial}{\partial t}$ is Killing, we do not assume that $\delta \tau^{i}{ }_{0}=0$. We do not assume that $\Delta^{a}{ }_{b}$ has the same symmetries as the unperturbed metric.
} 
Now

$$
\begin{aligned}
N\left(\delta \widetilde{\omega}_{i}^{j}\right)_{j}-\left(\partial_{j} N\right) \Delta_{i}{ }^{j}= & N\left(\widetilde{D}_{j} S_{i}{ }^{j}-\partial_{i} S\right) \\
& -\widetilde{D}_{j}\left(N A_{i}{ }^{j}\right)-\left(\partial_{j} N\right) S_{i}{ }^{j}
\end{aligned}
$$

and $\Omega\left[e^{a}, \mathcal{L}_{\vec{K}} e^{a}, \delta e^{a}\right]$ should be gauge invariant and hence independent of $A_{i}{ }^{j}$. We might expect

$$
\int_{\partial \Sigma_{t}} \widetilde{D}_{j}\left(N A_{i}{ }^{j}\right) * e^{0 i}=0
$$

as it is the integral of a divergence on $\partial \Sigma_{t}$ (the integral over $* e^{0 i}$ forces $e^{i}$ to be normal to $\partial \Sigma_{t}$, and hence $j$ is restricted to be a tangential index). This can be proven more rigorously using a straightforward application of Frobenius' theorem but we omit the details. Invoking (B28) Eq. (B27) can be rearranged to

$$
\begin{aligned}
& \Omega\left[e^{a}, \mathcal{L}_{\vec{K}} e^{a}, \delta e^{a}\right] \\
& =\frac{1}{8 \pi} \int_{\partial \Sigma_{t}}\left\{N\left(\widetilde{D}_{j} S_{i}{ }^{j}-\partial_{i} S\right)+\left(\partial_{i} N\right) S-\left(\partial_{j} N\right) S_{i}{ }^{j}\right. \\
& \left.\quad+X_{i j} N^{j}-N_{i}\left(\kappa_{j k} S^{j k}+\delta \kappa\right)\right\} * e^{0 i} .
\end{aligned}
$$

Assuming that $\Sigma_{t}$ can be foliated into two-dimensional surfaces $\phi_{t, r}$, parametrized by $r$, we have

$$
\begin{aligned}
& \Phi\left[e^{a}, \mathcal{L}_{\vec{K}} e^{a}, \delta e^{a}\right] \\
& =\frac{1}{8 \pi} \int_{\sigma_{t, r}}\left\{N\left(\widetilde{D}_{j} S_{i}{ }^{j}-\partial_{i} S\right)+\left(\partial_{i} N\right) S-\left(\partial_{j} N\right) S_{i}{ }^{j}\right. \\
& \left.\quad+X_{i j} N^{j}-N_{i}\left(\kappa_{j k} S^{j k}+\delta \kappa\right)\right\} * e^{0 i}
\end{aligned}
$$

is guaranteed to be independent of $t$ and $r$ if $\vec{K}=\frac{\partial}{\partial t}$ is Killing.

\section{APPENDIX C: CONNECTION 1-FORMS IN BONDI-SACHS GAUGE}

We list here the connection 1-forms for asymptotically flat metrics in Bondi-Sachs the gauge as defined in (50) and (51) in the text. First write

$$
f=U \exp W \quad \text { and } \quad g=U^{-1} \exp (W)
$$

with $f(u, r, \vartheta, \varphi)$ and $g(u, r, \vartheta, \varphi)$ tending to one as $r \rightarrow \infty$ in terms of which

$$
e^{0}=f d u+g d r, \quad e^{1}=g d r .
$$

We also have

$$
e^{i}=r \widetilde{e}^{i}-V^{i} d u
$$

with $\widetilde{e}^{i}(i=2,3)$ orthonormal 1-forms for the 2-sphere metric with $r$ and $u$ constant. In terms of the round unit metric on the 2-sphere it is convenient to choose a gauge in which

$$
\widetilde{e}^{i}=C^{i} \widehat{e}^{i}
$$

with

$$
C_{j}^{i}=\left(\begin{array}{cc}
e^{\gamma} \cosh \delta & e^{-\gamma} \sinh \delta \\
e^{\gamma} \sinh \delta & e^{-\gamma} \cosh \delta
\end{array}\right)
$$

and

$$
\hat{e}^{2}=d \vartheta, \quad \hat{e}^{3}=\sin \vartheta d \varphi,
$$

$\operatorname{det} C=1$, but we shall not need this explicit form.

The connection 1-forms arising from (C1) and (C2) can be calculated from (A1), they are ${ }^{16}$

$$
\begin{aligned}
\omega_{01}= & \frac{1}{f g}\left(\dot{g}+V^{i} \partial_{i} g\right)\left(e^{0}-e^{1}\right)-\frac{f^{\prime}}{f g} e^{0} \\
& -\frac{1}{2}\left(\frac{\partial_{i} f}{f}-\frac{\partial_{i} g}{g}+\frac{V_{i}^{\prime}}{f g}\right) e^{i}, \\
\omega_{0 i}= & -\frac{\partial_{i} f}{f} e^{0}+\frac{1}{2}\left(\frac{\partial_{i} f}{f}-\frac{\partial_{i} g}{g}-\frac{V_{i}^{\prime}}{f g}\right) e^{1} \\
& -\frac{1}{f}\left(\widetilde{D}_{\{i} V_{j\}}+\tau_{\{i j\}}\right) e^{j}, \\
\omega_{1 i}= & \frac{\partial_{i} g}{g} e^{1}+\frac{1}{2}\left(\frac{\partial_{i} f}{f}-\frac{\partial_{i} g}{g}+\frac{V_{i}^{\prime}}{f g}\right) e^{0} \\
& +\left(\frac{1}{f}\left(\widetilde{D}_{\{i} V_{j\}}+\tau_{\{i j\}}\right)-\frac{1}{g}\left(\frac{\delta_{i j}}{r}+\rho_{\{i j\}}\right)\right) e^{j}, \\
\omega_{i j}= & \frac{1}{r} \widetilde{\omega}_{i j}+\frac{1}{f}\left(\widetilde{D}_{[i} V_{j]}-\tau_{[i j]}\right)\left(e^{0}-e^{1}\right)-\frac{1}{g} \rho_{[i j]} e^{1},
\end{aligned}
$$

where $\doteq \partial_{u},{ }^{\prime}=\partial_{r}, \partial_{i}=\left(\widetilde{e}^{-1}\right)^{\alpha}{ }_{i} \partial_{\alpha}$ and

$$
\rho_{j}^{i}=\left(C^{\prime} C^{-1}\right)_{j}^{i}
$$

$\widetilde{\omega}_{i j}=\widetilde{\omega}_{i j, k} \widetilde{e}^{k}$ are the connection 1-forms associated with $\widetilde{e}^{i}$ on the 2-sphere and $\widetilde{D}_{i}$ the associated covariant derivative.

With $f=U e^{W}$ and $g=U^{-1} e^{W}$ these expressions are used to calculate $\Phi$ in Sec. IVA 3.

Note that we have not assumed any symmetries, in particular it has not been assumed that $\vec{K}=\partial_{t}$ is Killing. When $\partial_{t}$ is Killing $\dot{g}$ and $\tau_{\{i j\}}$ are zero, but in any case these do not appear in $\Phi(\vec{K})$ at large $r$.

\footnotetext{
${ }^{16}\{i j\}$ denotes symmetrization, with normalization $\frac{1}{2}(i j+j i) ;[i j]$ denotes antisymmetrization, with normalization $\frac{1}{2}(i j-j i)$
} 
[1] C. Crnković and E. Witten, Covariant Description of Canonical Formalism in Geometrical Theories, in Three Hundred Years of Gravitation, edited by W. Isreal and S. W. Hawking (Cambridge University Press, Cambridge, England, 1987).

[2] J. L. Friedman, Commun. Math. Phys. 62, 247 (1978).

[3] J. Lee and R. M. Wald, J. Math. Phys. 31, 725 (1990).

[4] R. M. Wald, J. Math. Phys. 31, 2378 (1990).

[5] R. M. Wald, Phys. Rev. D 48, R3427 (1993).

[6] V. Iyer and R. M. Wald, Phys. Rev. D 50, 846 (1994).

[7] A. Bilal, arXiv:0802.0634.

[8] B. P. Dolan, preceding Paper, Phys. Rev. D 98, 044009 (2018).

[9] R. Arnowitt, S. Deser, and C. W. Misner, Phys. Rev. 117, 1595 (1960).

[10] A. Komar, Phys. Rev. 113, 934 (1959).

[11] J. Katz, Classical Quantum Gravity 2, 423 (1985).
[12] K. Hajian and M. M. Sheikh-Jabbari, Phys. Rev. D 93, 044074 (2016).

[13] I. Papadimitriou and K. Skenderis, J. High Energy Phys. 08 (2005) 004.

[14] M. Henneaux and C. Teitelboim, Commun. Math. Phys. 98, 391 (1985).

[15] G. W. Gibbons, M. J. Perry, and C. N. Pope, Classical Quantum Gravity 22, 1503 (2005).

[16] J. D. Brown and J. W. York, Jr., Phys. Rev. D 47, 1407 (1993).

[17] H. Bondi, Nature (London) 186, 535 (1960).

[18] R. Bott and L.W. Tu, Differential Forms in Algebraic Topology, Graduate Texts in Mathematics, Vol. 82 (Springer, New York, 1982).

[19] S. W. Hawking and G. T. Horowitz, Classical Quantum Gravity 13, 1487 (1996).

[20] W. Simon, Gen. Relativ. Gravit. 17, 439 (1985).

[21] R. Sachs, Phys. Rev. 128, 2851 (1962). 